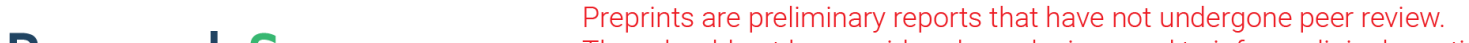 They should not be considered conclusive, used to inform clinical practice, or referenced by the media as validated information. \\ COVID-19 mortality analysis from soft- data multivariate curve regression and machine learning
}

\section{Antoni Torres-Signes}

University of Malaga: Universidad de Malaga

\section{Pilar Frías}

University of Jaen: Universidad de Jaen

Maria Dolores Ruiz-Medina ( $\Delta$ mruiz@ugr.es )

University of Granada https://orcid.org/0000-0001-7445-7060

\section{Research Article}

Keywords: COVID-19 analysis, Curve regression, Hard-data, Machine Learning, Multivariate time series, Soft-data

Posted Date: March 29th, 2021

DOl: https://doi.org/10.21203/rs.3.rs-158858/v1

License: (c) (i) This work is licensed under a Creative Commons Attribution 4.0 International License. Read Full License

Version of Record: A version of this preprint was published at Stochastic Environmental Research and Risk Assessment on April 19th, 2021. See the published version at https://doi.org/10.1007/s00477-02102021-0. 


\title{
COVID-19 mortality analysis from soft-data multivariate curve regression and machine learning
}

\author{
Antoni Torres-Signes · María P. Frías . \\ María D. Ruiz-Medina
}

Received: date / Accepted: date

\begin{abstract}
A multiple objective space-time forecasting approach is presented involving cyclical curve log-regression, and multivariate time series spatial residual correlation analysis. Specifically, the mean quadratic loss function is minimized in the framework of trigonometric regression. While, in our subsequent spatial residual correlation analysis, maximization of the likelihood allows us to compute the posterior mode in a Bayesian multivariate time series soft-data framework. The presented approach is applied to the analysis of COVID-19 mortality in the first wave affecting the Spanish Communities, since March, 8, 2020 until May, 13, 2020. An empirical comparative study with Machine Learning (ML) regression, based on random $k$-fold cross-validation, and bootstrapping confidence interval and probability density estimation, is carried out. This empirical analysis also investigates the performance of ML regression models in a hard- and soft- data frameworks. The results could be extrapolated to other counts, countries, and posterior COVID-19 waves.
\end{abstract}

Key words. COVID-19 analysis; Curve regression; Hard-data; Machine Learning; Multivariate time series; Soft-data

Mathematics Subject Classification (2010) MSC code1 62F40 - 62F15 and $62 \mathrm{~F} 10 \cdot 90 \mathrm{~B} 99$

A. Torres-Signes

Department of Statistics and Operation Research, Faculty of Sciences, University of Málaga, Spain

E-mail: atisignes@uma.es

M. P. Frías

Department of Statistics and Operation Research, Faculty of Sciences, University of Jaén, Spain

E-mail: mpfrias@ujaen.es

M.D. Ruiz-Medina

Department of Statistics and Operation Research, Faculty of Sciences, University of Granada, Spain

E-mail: mruiz@ugr.es 


\section{Introduction}

Coronavirus disease 2019 (COVID-19) rapidly spreads around many other countries, since December 2019 when arises in China (see [50]; [57]; [65]). The effective allocation of medical resources requires the derivation of predictive techniques, describing the spatiotemporal dynamics of COVID-19 (see, e.g., [17]; [32]; [41]; [45], just to mention a few). Epidemiological models can contribute to the analysis of the causes, dynamics, and spread of this pandemic (see, e.g, [24]; [30]; [35], and the references therein). Short-term forecasts can be obtained adopting the framework of compartmental SIR (susceptibleinfectious-recovered) models, based on ordinary differential equations (see, e.g. [6] [19]; [28]; [31]; [34]; [39]; [43]; [54]; [61]; [63]). An extensive literature is available, including different versions of compartmental models, like SIRsusceptible (SIRS, [18]), and delay differential equation based formulations (see [8]; [38]; [49]). Spatial extensions, based on reaction-diffusion models, reflecting the infectious disease spread over a spatial region can be found, for instance, in [21] and [59]. SEIRD (susceptible, exposed, infected, recovered, deceased) models, incorporating the spatial spread of the disease with inhomogeneous diffusion terms are also analyzed (see [47] and [48]). The stochastic version of SIR-type models intends to cover several limitations detected regarding uncertainly, in the observations, and the hidden dynamical epidemic process. Markov chain SIR based modelling (see [5]; [60]), and some recent stochastic formulations involving complex networks (see [64]; [56]) or drugresistant influenza (see [13]) constitute some alternatives. A Bayesian hierarchical statistical SIRS model framework is adopted in [1]; [2]; [5]; [20] taking into account the observation error in the counts, and uncertainty in the parameter space. Beyond SIR modeling, we mention here the multivariate and survival analysis based approaches to modelling, for instance, infection, incubation and recovering random periods, affecting the containment of COVID-19 (see, e.g., [10] ; [29]; [42]; [58]).

In a first stage, most of the above referred models have been adapted and applied to approximate the space/time evolution of COVID-19 incidence and mortality. That is the case, for instance, of the three models presented in [46], which were validated with outbreaks of other diseases different from COVID19. Alternative SEIR type models, involving stochastic components, are formulated in [33]. A revised SEIR model has also been proposed in [62] (see also [23]). A $\theta$-SEIHRD model, able to estimate the number of cases, deaths, and needs of beds in hospitals, is introduced in [26], adapted to COVID-19, based on the Be-CoDiS model (see [27]). Due to the low quality of the records available, and the hidden sample information, the most remarkable feature in this research area is the balance between complexity and indentifiability of model parameters. Recently, an attempt to simplify modelling strategies, applied to COVID-19 data analysis, is presented in [44], in terms of $\theta$-SEIHQRD model. Mitigation of undersampling is proposed in [36], based on re-scaling of statistics involved in the epidemic properties analysis, like mortality analysis, useful between countries with similar levels of health care. 
Nowadays ML models have established themselves as serious contenders to classical statistical models in the area of forecasting. Research started in the eighties with the development of the neural network model. Subsequently, research extended this concept to alternative models, such as support vector machines, decision trees, and others (see, e.g., [4]; [9]; [22]; [40]). In general, curve regression techniques based on a function basis, usually in the space of square integrable functions with respect to a suitable probability measure, allow short- and long- term forecast. Thus, depending on our choice of the function basis, and the probability measure selected, particle and field views could be combined. Note that the classical stochastic diffusion models offer a particle rather than a field view (see, e.g., [37]).

Linear regression, multilayer perceptron and vector autoregression methods have been applied in [51] to predicting COVID-19 spread, and anticipating the potential patterns of COVID-19 effects (see also Section 2 of [51], on related work). Early stage location of COVID-19 is addressed in [7], applying machine learning strategies actualized on stomach Computed Tomography pictures. [15] evaluates association between meteorological factors and COVID-19 spread. They concluded that average temperature, minimum relative humidity, and precipitation were better predictors, displaying possible non-linear correlations with COVID-19 variables. These conclusions are crucial in the subsequent machine learning regression based analysis.

This paper presents a multiple objective space-time forecasting approach, where curve trigonometric log-regression is combined with multivariate time series spatial residual analysis. In our curve regression model fitting, we are interested on reflecting the cyclical behavior of COVID-19 mortality induced by the hardening or relaxation of the containment measures, adopted to mitigate the increase of infections and mortality. The trigonometric basis (sines and cosines) is then selected in our spatial heterogeneous curve log-regression model fitting. The ratio of the expected minimized empirical risk, and the corresponding expected value of the quadratic loss function at such a minimizer is considered for model selection (see, e.g., [14]). Note that this selection procedure provides an agreement between the expected minimum empirical risk, and the corresponding expected theoretical loss function value.

The penalized factor proposed in [14], applied to our choice of the truncation parameter, leads to the dimension of the subspace, where our curve regression estimator is approximated, at any spatial location. This model selection procedure is asymptotically equivalent to Akaike correction factor. A robust modification of the Akaike information criterion can be found, for example, in [3]. As an alternative, one can consider cross-validation criterion for selecting the best subset of explanatory variables (see [53], where a mixedinteger optimization approach is proposed in this context).

Beyond asymptotic analysis, model selection from finite sample sizes constitutes a challenging topic in our approach. Here, we compute a bootstrap estimator of the expected generalization error from the expected training error. Bootstrap confidence intervals are provided for the spatial mean of the curve regression predictor, and for the expected training error of the curve re- 
gression, and the multivariate time-series residual predictors. The bootstrap probability distribution of these statistics is also computed.

In our multivariate time series analysis of the regression residuals, a classical and Bayesian componentwise estimation of the spatial linear correlation is achieved. The presented multiple objective forecasting approach is applied to the spatiotemporal analysis of COVID-19 mortality in the first wave affecting the Spanish Communities, since March, 8, 2020 until May, 13, 2020. This approach takes into account the observation error in the counts, and uncertainty in the parameter space and hidden pandemic process. Our results show a remarkable qualitative agreement with the reported epidemiological data.

It is well-known that the limited availability of hard-data affects spacetime analysis and, hence, the incorporation of soft-data into ML regression models can help this analysis, providing a global view of the available sample information (see, e.g., [16]). In the empirical comparative analysis carried out, involving ML regression models, and the presented approach, input hard- and soft-data are considered. This analysis is based on cross-validation, and bootstrapping confidence intervals and probability density estimation. Specifically, random $k$-fold $(k=5,10)$ cross-validation, in terms of Symmetric Mean Absolute Percentage Errors (SMAPEs), evaluates the performance of the compared regression models from hard- and soft-data. The bootstrap confidence intervals, and the probability density estimates of the spatially averaged SMAPEs provide another view of this empirical comparative analysis, supporting the previous random $k$-fold based model classification. In the soft-data category, where our approach is compared with ML regression, short-term spatial correlations are also incorporated.

We can conclude that almost the best performance in both, hard- and softdata categories, is displayed by Radial Basis Function Neural Network (RBF), and Gaussian Processes (GP). Both approaches are improved, when soft-data are incorporated into the regression analysis. Slightly differences are observed in the performance of Support Vector Regression (SVR) and Bayesian Neural Networks (BNN). Multilayer Perceptron (MLP) gets over GRNN, presenting better estimation results when hard-data are analyzed. Our trigonometric curve regression, and classical, based on empirical moments, multivariate time series residual analysis presents similar SMAPEs to Generalized Regression Neural Network (GRNN), in relation to random $k$-fold and bootstrapping distributional characteristics, displaying the worst performance in the softdata category. Note that, GRNN is also favored by the soft-data category. In this category, BNN and our approach show very similar performance, when trigonometric regression is combined with Bayesian multivariate time series residual prediction. Indeed, some slightly better bootstrapping distributional characteristics of our approach respect to BNN are observed in the soft-data category.

The outline of the paper is the following. The modeling approach is introduced in Section 2. Section 3 describes the multiple objective forecasting methodology. This methodology is applied to the spatiotemporal statistical analysis of COVID-19 mortality in Spain in Section 4. The empirical compar- 
ative study with ML regression models is given in Section 5. Conclusions about our data-driven model ranking can be found in Section 6. In the Supplementary Material, a brief introduction to our implementation of ML models from hard- and soft-data is provided. Additional information about our empirical comparative study is also displayed. In particular, the observed and predicted mortality cumulative cases, and log-risk curves are displayed.

\section{Data model}

Let $(\Omega, \mathcal{A}, \mathcal{P})$ be the basic probability space. Consider $H=L^{2}\left(\mathbb{R}^{d}\right), d \geq 2$, the space of square-integrable functions on $\mathbb{R}^{d}$, to be the underlying real separable Hilbert space. In the following, we denote by $\mathcal{B}^{d}$ the Borel $\sigma$-algebra in $\mathbb{R}^{d}$, $d \geq 1$. Let $X=\left\{X_{t}(\mathbf{z}), \mathbf{z} \in \mathbb{R}^{d}, t \in \mathbb{R}_{+}\right\}$be our spatiotemporal input harddata process on $(\Omega, \mathcal{A}, \mathcal{P})$, satisfying $E\left[\left\|X_{t}(\cdot)\right\|_{H}^{2}\right]<\infty$, for any time $t \in \mathbb{R}_{+}$. The input soft-data process over any spatial bounded set $D \in \mathcal{B}^{d}$ is then defined as

$$
\left\{X_{t}(h)=\int_{D} X_{t}(\mathbf{z}) h(\mathbf{z}) d \mathbf{z}, h \in \mathcal{C}_{0}^{\infty}(D), t \in \mathbb{R}_{+}\right\},
$$

where $\mathcal{C}_{0}^{\infty}(D)$ denotes the space of infinite differentiable functions, with compact support contained in $D$. For each bounded set $D \in \mathcal{B}^{d}$, define

$$
\Lambda=\left\{\Lambda_{t}(h)=\exp \left(X_{t}(h)\right), h \in \mathcal{C}_{0}^{\infty}(D), t \in \mathbb{R}_{+}\right\}
$$

Assume that, for any finite positive interval $\mathcal{T} \in \mathcal{B}$, and bounded set $D \in \mathcal{B}^{d}$,

$$
\mathcal{I}_{\mathcal{T}}(h)=\int_{\mathcal{T}} \exp \left(X_{t}(h)\right) d t<\infty, \quad \forall h \in \mathcal{C}_{0}^{\infty}(D),
$$

almost surely (a.s.). Let $\left\{N_{h}:(\Omega, \mathcal{A}, \mathcal{P}) \times \mathcal{B} \longrightarrow \mathbb{N}, h \in H\right\}$ be a family of random counting measures. Assume that, for $h \in \mathcal{C}_{0}^{\infty}(D)$, with $D \in \mathcal{B}^{d}$, a bounded set, given the observation $\left\{x_{t}(h), t \in \mathcal{T}\right\}$, at the finite temporal interval $\mathcal{T} \in \mathcal{B}$, of the input soft-data process over the spatial $h$-window in $D$, the conditional probability distribution of the number of random events $N_{h}(\mathcal{T})$, that occur in $\mathcal{T} \in \mathcal{B}$, is a Poisson probability distribution with mean $\int_{\mathcal{T}} \exp \left(x_{t}(h)\right) d t$. We refer to $\mathcal{I}_{\mathcal{T}}(h)$ as the generalized cumulative mortality risk random process over the interval $\mathcal{T}$. Hence, the input hard-data process $X=\left\{X_{t}(\mathbf{z}), \mathbf{z} \in \mathbb{R}^{d}, t \in \mathbb{R}_{+}\right\}$defines the spatiotemporal mortality log-risk process.

The following curve regression model is fitted from the input soft-data process, for $p=1, \ldots, P$,

$$
\begin{aligned}
& \ln \left(\Lambda_{t}\right)\left(\psi_{p, \varpi_{p}}\right)=g_{t}\left(\psi_{p, \varpi_{p}}, \boldsymbol{\theta}(p)\right)+\varepsilon_{t}\left(\psi_{p, \varpi_{p}}\right) \\
& \quad=\left\langle g_{t}(\cdot, \boldsymbol{\theta}(p)), \psi_{p, \varpi_{p}}(\cdot)\right\rangle_{H}+\left\langle\varepsilon_{t}(\cdot), \psi_{p, \varpi_{p}}(\cdot)\right\rangle_{H}, t \in \mathbb{R}_{+},
\end{aligned}
$$


where

$$
\begin{aligned}
g_{t}\left(\psi_{p, \varpi_{p}}, \boldsymbol{\theta}(p)\right) & =\int_{\mathcal{D}_{p}} g_{t}(\mathbf{z}, \boldsymbol{\theta}(p)) \psi_{p, \varpi_{p}}(\mathbf{z}) d \mathbf{z} \\
\langle f, g\rangle_{H} & =\int_{\mathbb{R}^{d}} f(\mathbf{z}) g(\mathbf{z}) d \mathbf{z},
\end{aligned}
$$

with $\left\{\psi_{p, \varpi_{p}}, p=1, \ldots, P\right\} \subset H$ denoting a function family in $H$, whose elements have respective compact supports $\mathcal{D}_{p}, p=1, \ldots, P$, defining the $p$ small-areas, where the counts are aggregated, satisfying suitable regularity conditions. For each $p=1, \ldots, P$, the vector $\varpi_{p}$ contains the center and bandwidth parameters, defining the window selected in the analysis of the smallarea $p$. For each $p \in\{1, \ldots, P\}, \boldsymbol{\theta}(p)=\left(\theta^{1}(p), \ldots, \theta^{q}(p)\right) \in \Theta$ represents the unknown parameter vector to be estimated at the $p$ region, and $\Theta$ is the open set defining the parameter space, whose closure $\Theta^{c}$ is a compact set in $\mathbb{R}^{q}$. We assume that $g_{t}$ is of the form (see, e.g., [25])

$g_{t}(\boldsymbol{\theta}(p))=\sum_{k=1}^{N}\left(A_{k}(p) \cos \left(\varphi_{k}(p) t\right)+B_{k}(p) \sin \left(\varphi_{k}(p) t\right)\right), p=1, \ldots, P, \quad t \in \mathbb{R}_{+}$,

whose spatial-dependent parameters are given by the temporal scalings $\left(\varphi_{1}(\cdot), \ldots, \varphi_{N}(\cdot)\right)$, and Fourier coefficients $\left(A_{1}(\cdot), B_{1}(\cdot), \ldots, A_{N}(\cdot), B_{N}(\cdot)\right)$. For simplifications purposes, we will consider that the scaling parameters $\varphi_{k}$, $k=1, \ldots, N$, are known, and fixed over the $P$ spatial regions. Also, $C_{k}^{2}(\cdot)=$ $A_{k}^{2}(\cdot)+B_{k}^{2}(\cdot)>0$, for $k=1, \ldots, N$, where $N$ denotes the truncation parameter, that will be selected according to the penalized factor proposed in [14], as we explain in more detail in Section 3. Thus,

$$
\boldsymbol{\theta}(p)=\left(A_{1}(p), B_{1}(p), \ldots, A_{N}(p), B_{N}(p)\right), \quad p=1, \ldots, P .
$$

To analyze the spatial correlation between regions, a multivariate autoregressive model is considered for prediction of the regression residual term at each region $p \in\{1, \ldots, P\}$. Particularly, for any $T \geq 2, \varepsilon_{t}$ in equation $(3)$ is assumed to satisfy the state equation, for $p=1, \ldots, P$,

$$
\varepsilon_{t}\left(\psi_{p, \varpi_{p}}\right)=\sum_{q=1}^{P} \rho\left(\psi_{q, \varpi_{q}}\right)\left(\psi_{p, \varpi_{p}}\right) \varepsilon_{t-1}\left(\psi_{q, \varpi_{q}}\right)+\nu_{t}\left(\psi_{p, \varpi_{p}}\right),
$$

where, for any $t \in \mathbb{R}_{+}$, and $p, q=1, \ldots, P$,

$$
\begin{aligned}
\varepsilon_{t}\left(\psi_{p, \varpi_{p}}\right) & =\int_{\mathcal{D}_{p}} \varepsilon_{t}(\mathbf{z}) \psi_{p, \varpi_{p}}(\mathbf{z}) d \mathbf{z} \\
\nu_{t}\left(\psi_{p, \varpi_{p}}\right) & =\int_{\mathcal{D}_{p}} \nu_{t}(\mathbf{z}) \psi_{p, \varpi_{p}}(\mathbf{z}) d \mathbf{z} \\
\rho\left(\psi_{q, \varpi_{q}}\right)\left(\psi_{p, \varpi_{p}}\right) & =\int_{\mathcal{D}_{p} \times \mathcal{D}_{p}} \rho(\mathbf{z}, \mathbf{y}) \psi_{p, \varpi_{p}}(\mathbf{z}) \psi_{q, \varpi_{q}}(\mathbf{y}) d \mathbf{y} d \mathbf{z} .
\end{aligned}
$$


Here, $\left(\nu_{t}\left(\psi_{p, \varpi_{p}}\right), p=1, \ldots, P\right), t \in \mathbb{R}_{+}$, are assumed to be independent zeromean Gaussian $P$-dimensional vectors. For $p, q \in\{1, \ldots P\}$, the projection $\rho\left(\psi_{p, \varpi_{p}}\right)\left(\psi_{q, \varpi_{q}}\right)$ then keeps the temporal linear autocorrelation at each spatial region for $p=q$, and the temporal linear cross-correlation between regions for $p \neq q$ of the regression error $\left\{\varepsilon_{t}(\cdot), t \in \mathbb{R}_{+}\right\}$(see, [11]).

\section{Implementation of the curve regression model and spatial residual analysis}

Let $\mathcal{D}_{1}, \ldots, \mathcal{D}_{P}$ be the small-areas, where the counts are aggregated, and $\left\{\psi_{p, \varpi_{p}}, \varpi_{p}=\left(c_{p}, \rho_{p}\right), p=1, \ldots, P\right\} \subset H$ be the functions with respective compact supports $\mathcal{D}_{1}, \ldots, \mathcal{D}_{P}$. Particularly, we denote by $c_{p}, p=1, \ldots, P$, the centers respectively allocated at the regions $\mathcal{D}_{1}, \ldots, \mathcal{D}_{P}$, and by $\rho_{1}, \ldots, \rho_{P}$, the bandwidth parameters providing the associated window sizes.

In practice, from the observation model (3), to find $g_{t}$ in (5) minimizing the expected quadratic loss function, or expected risk, we look for the minimizer $\widehat{\boldsymbol{\theta}}_{T}(p)$ of the empirical regression risk

$$
L_{T}\left(\widehat{\boldsymbol{\theta}}_{T}(p)\right)=\inf _{\boldsymbol{\theta}(p) \in \Theta^{c}} L_{T}(\boldsymbol{\theta}(p))=\inf _{\boldsymbol{\theta}(p) \in \Theta^{c}} \frac{1}{T} \sum_{t=1}^{T}\left|\ln \left(\Lambda_{t}\right)\left(\psi_{p, \varpi_{p}}\right)-g_{t}(\boldsymbol{\theta}(p))\right|^{2} .
$$

Truncation parameter $N$ is then selected to controlling the ratio between the expected quadratic loss function at $\widehat{\boldsymbol{\theta}}_{T}(p)$, and the expected value of the minimized empirical risk from the identity

$$
\begin{aligned}
& E\left[\ln \left(\Lambda_{t}\right)\left(\psi_{p, \varpi_{p}}\right)-g_{t}\left(\psi_{p, \varpi_{p}}, \widehat{\boldsymbol{\theta}}_{T}(p)\right]^{2}\right. \\
& =E\left[L_{T}\left(\widehat{\boldsymbol{\theta}}_{T}(p)\right)\right]\left(1-\frac{N}{T}\right)^{-1}\left(1+\frac{\sum_{i=1}^{N} 1 / \lambda_{i}}{T}\right),
\end{aligned}
$$

where, for $i=1, \ldots, N, 1 / \lambda_{i}$ denotes the inverse of the $i$ th eigenvalue of the matrix $\Phi^{T} \Phi$, with $\Phi$ being a $T \times N$ matrix, whose elements are the values of the $N$ trigonometric basis functions selected at the time points $t=1, \ldots, T$. Parameter $N$ should be such that $N<<T$. Note that, asymptotically, when $N \rightarrow \infty, \Phi^{T} \Phi$ goes to the identity matrix, and for $i=1, \ldots, N, 1 / \lambda_{i} \sim 1$. For $p=1, \ldots, P$, we have considered the minimized empirical risk

$$
L_{T}\left(\widehat{\boldsymbol{\theta}}_{T}(p)\right)=\frac{1}{T} \widetilde{\mathcal{R}}^{T}(p)\left(I_{T \times T}-\Phi\left(\Phi^{T} \Phi\right)^{-1} \Phi^{T}\right) \widetilde{\mathcal{R}}(p),
$$

with, for $p=1, \ldots, P$,

$$
\widetilde{\mathcal{R}}(p)=\left(\sum_{k=N+1}^{\infty}\left(A_{k}(p) \cos \left(\varphi_{k} t\right)+B_{k}(p) \sin \left(\varphi_{k} t\right)\right)+\varepsilon_{t}\left(\psi_{p, \varpi_{p}}\right), t=1, \ldots, T\right) .
$$


Thus, our regression predictor is given by

$$
\widehat{\ln \left(\Lambda_{t}\right)}\left(\psi_{p, \varpi_{p}}\right)=g_{t}\left(\widehat{\boldsymbol{\theta}}_{T}(p)\right), \quad p=1, \ldots, P
$$

(see Theorem 1 in [25] about conditions for the weak-consistency of (10)).

The regression residuals

$$
\mathbf{Y}=\left\{Y_{t}\left(\psi_{p, \varpi_{p}}\right)=\ln \left(\Lambda_{t}\right)\left(\psi_{p, \varpi_{p}}\right)-g_{t}\left(\widehat{\boldsymbol{\theta}}_{T}(p)\right), t=1, \ldots, T, p=1, \ldots, P\right\}
$$

and the empirical nuclear autocovariance and cross-covariance operators

$$
\begin{array}{r}
\widehat{R}_{0, T}^{\mathbf{Y}}\left(\psi_{p, \varpi_{p}}\right)\left(\psi_{q, \varpi_{q}}\right)=\frac{1}{T} \sum_{t=1}^{T} Y_{t}\left(\psi_{p, \varpi_{p}}\right) Y_{t}\left(\psi_{q, \varpi_{q}}\right) \\
\widehat{R}_{1, T}^{\mathbf{Y}}\left(\psi_{p, \varpi_{p}}\right)\left(\psi_{q, \varpi_{q}}\right)=\frac{1}{T-1} \sum_{t=1}^{T-1} Y_{t}\left(\psi_{q, \varpi_{q}}\right) Y_{t+1}\left(\psi_{p, \varpi_{p}}\right), p, q=1, \ldots, P,
\end{array}
$$

will be considered in the estimation of the spatial linear residual correlation (see [11]). A truncation parameter $k(T)$ is also considered here to remove the ill-posed nature of this estimation problem. Particularly, $k(T)$ must satisfy $k(T) \rightarrow \infty, k(T) / T \rightarrow 0, T \rightarrow \infty$. A suitable choice of $k(T)$ also ensures strong-consistency of the estimator

$$
\widehat{\rho}_{k(T)}\left(\psi_{p, \varpi_{p}}\right)\left(\psi_{q, \varpi_{q}}\right)=\sum_{k, l=1}^{k(T)} \frac{\left\langle\psi_{p, \varpi_{p}}, \phi_{k, T}\right\rangle_{H}\left\langle\psi_{q, \varpi_{q}}, \phi_{l, T}\right\rangle_{H}}{\lambda_{k, T}\left(\widehat{R}_{0, T}^{\mathbf{Y}}\right)} \widehat{R}_{1, T}^{\mathbf{Y}}\left(\phi_{k, T}\right)\left(\phi_{l, T}\right),
$$

for $p, q=1, \ldots, P$ (see [11]). Here,

$$
\widehat{R}_{0, T}^{\mathbf{Y}}=\sum_{k=1}^{T} \lambda_{k, T}\left(\widehat{R}_{0, T}^{\mathbf{Y}}\right)\left[\phi_{k, T} \otimes \phi_{k, T}\right]
$$

where $\left\{\lambda_{k, T}\left(\widehat{R}_{0, T}^{\mathbf{Y}}\right), \quad k=1, \ldots, T\right\}$ and $\left\{\phi_{k, T}, k \geq 1\right\}$ denote the empirical eigenvalues and eigenvectors of $\widehat{R}_{0, T}^{\mathbf{Y}}$, respectively. Particularly, we consider $k(T)=\ln (T)$ (see [11]). The classical plug-in predictor is then computed, for each $p=1, \ldots, P$, as

$$
\widehat{Y}_{t}^{k(T)}\left(\psi_{p, \varpi_{p}}\right)=\sum_{q=1}^{P} \widehat{\rho}_{k(T)}\left(\psi_{q, \varpi_{q}}\right)\left(\psi_{p, \varpi_{p}}\right) Y_{t-1}\left(\psi_{q, \varpi_{q}}\right), \quad t \geq 1 .
$$

Under the Gaussian distribution of $\nu_{t}$, in the Bayesian estimation of $\rho$, from (6), the likelihood function, defining the objective function, is given by, 
for each $p=1, \ldots, P$,

$$
\begin{gathered}
\widetilde{L}_{p}\left(\varepsilon_{1 p}, \ldots, \varepsilon_{T p}, \varepsilon_{0 q}, \ldots, \varepsilon_{(T-1) q} \rho\left(\psi_{q, \varpi_{q}}\right)\left(\psi_{p, \varpi_{p}}\right), q=1, \ldots, P\right) \\
=\frac{\exp \left(-\frac{1}{2 \sigma_{p}^{2}} \sum_{t=1}^{T}\left(\varepsilon_{t}\left(\psi_{p, \varpi_{p}}\right)-\sum_{q=1}^{P} \varepsilon_{t-1}\left(\psi_{q, \varpi_{q}}\right) \rho\left(\psi_{q, \varpi_{q}}\right)\left(\psi_{p, \varpi_{p}}\right)\right)^{2}\right)}{\left(\sigma_{p} \sqrt{2 \pi}\right)^{T}} \\
\times \prod_{q=1}^{P}\left[\rho\left(\psi_{q, \varpi_{q}}\right)\left(\psi_{p, \varpi_{p}}\right)\right]^{a_{p q}-1}\left(1-\rho\left(\psi_{q, \varpi_{q}}\right)\left(\psi_{p, \varpi_{p}}\right)\right)^{b_{p q}-1} \\
\times \frac{\mathbb{I}_{\left\{0<\rho\left(\psi_{q, \varpi_{q}}\right)\left(\psi_{p, \varpi_{p}}\right)<1\right\}}}{\mathbb{B}\left(a_{p q}, b_{p q}\right)} \\
=\frac{1}{\left(\sigma_{p} \sqrt{2 \pi}\right)^{T}} \exp \left(-\frac{1}{2 \sigma_{p}^{2}} \sum_{t=1}^{T}\left[\nu_{t}\left(\psi_{p, \varpi_{p}}\right)\right]^{2}\right) \\
\times \prod_{q=1}^{P}\left[\rho\left(\psi_{q, \varpi_{q}}\right)\left(\psi_{p, \varpi_{p}}\right)\right]^{a_{p q}-1}\left(1-\rho\left(\psi_{q, \varpi_{q}}\right)\left(\psi_{p, \varpi_{p}}\right)\right)^{b_{p q}-1} \\
\times \frac{\mathbb{I}_{\left\{0<\rho\left(\psi_{q, \varpi_{q}}\right)\left(\psi_{p, \varpi_{p}}\right)<1\right\}}}{\mathbb{B}\left(a_{p q}, b_{p q}\right)}
\end{gathered}
$$

where, for each $p=1, \ldots, P$, the beta probability distributions with shape parameters $a_{p q}$ and $b_{p q}, q=1, \ldots, P$, respectively define the prior probability distributions of the independent random variables $\left\{\rho\left(\psi_{q, \varpi_{q}}\right)\left(\psi_{p, \varpi_{p}}\right), q=\right.$ $1, \ldots, P\}$. Here, for each $p=1, \ldots, P, \varepsilon_{t p}=\varepsilon_{t}\left(\psi_{p, \varpi_{p}}\right)=\left\langle\varepsilon_{t}, \psi_{p, \varpi_{p}}\right\rangle_{H}$, and $\sigma_{p}=\sqrt{E\left[\varepsilon_{t}\left(\psi_{p, \varpi_{p}}\right)\right]^{2}}$, for $t=0, \ldots, T$. As before, $\psi_{p, \varpi_{p}}$ weights the spatial sample information about the $p$ small-area, for $p=1, \ldots, P$. As usual, $\mathbb{I}_{0<\cdot<1}$ denotes the indicator function on the interval $(0,1)$, and $\mathbb{B}\left(a_{p q}, b_{p q}\right)$ is the beta function,

$$
\mathbb{B}\left(a_{p q}, b_{p q}\right)=\frac{\Gamma\left(a_{p q}\right) \Gamma\left(b_{p q}\right)}{\Gamma\left(a_{p q}+b_{p q}\right)} .
$$

From (15), the Bayesian predictor is obtained, for $p=1, \ldots, P$, as

$$
\widetilde{\varepsilon}_{t}\left(\psi_{p, \varpi_{p}}\right)=\sum_{q=1}^{P} \widetilde{\rho}\left(\psi_{q, \varpi_{q}}\right)\left(\psi_{p, \varpi_{p}}\right) \varepsilon_{t-1}\left(\psi_{q, \varpi_{q}}\right), \quad t \geq 1,
$$

with $\left(\widetilde{\rho}\left(\psi_{1, \varpi_{1}}\right)\left(\psi_{p, \varpi_{p}}\right), \ldots, \widetilde{\rho}\left(\psi_{P, \varpi_{P}}\right)\left(\psi_{p, \varpi_{p}}\right)\right)$ being computed by maximizing (15), to find the posterior mode (see [12], where Bayesian estimation is introduced in an infinite-dimensional framework). We refer to (16) as the Bayesian plug-in predictor of the residual mortality log-risk process at the $p$ small area, for $p=1, \ldots, P$. In practice, equation (15) is approximated from the computed values of the regression residual process. 


\section{Statistical analysis of COVID-19 mortality}

Our analysis is based on the daily records of COVID-19 mortality reported by the Spanish Statistical National Institute, since March, 8 to May, 13, 2020, at the 17 Spanish Communities. Interpolation at 265 temporal nodes, and cubic B-spline smoothing have been considered in the implementation of (3)-(5). The regression predictor is computed from equation (10), applying (7)-(8). Particularly,

$$
T(265,12)=\left(1-\frac{12}{265}\right)^{-1}\left(1+\frac{\sum_{i=1}^{N} 1 / \lambda_{i}}{T}\right)=1.1304,
$$

corresponds to our choice $N=6$ in (5). Tables $1-2$ below display the parameter estimates $\widehat{A}_{k}(\cdot)$ and $\widehat{B}_{k}(\cdot), k=1, \ldots, 6$, where $\varphi_{k}=\frac{2 \pi}{265}$ has been considered, for $k=1, \ldots, N=6$. In these tables and below, the following Spanish Community (SC) codes appear: C1 for Andalucía; C2 for Aragón; C3 for Asturias; C4 for Islas Baleares; C5 for Canarias; C6 for Cantabria; C7 for Castilla La Mancha; C8 for Castilla y León; C9 for Cataluña; C10 for Comunidad Valenciana; C11 for Extremadura; C12 for Galicia; C13 for Comunidad de Madrid; C14 for Murcia; C15 for Navarra; C16 for País Vasco, and C17 for La Rioja.

Table 1: Regression parameter estimates $\widehat{A}_{k}(\cdot), k=1, \ldots, 6$, at the 17 Spanish Communities

\begin{tabular}{|ccccccc|}
\hline SC $/ \mathbf{P E}$ & $\widehat{A}_{1}(\cdot)$ & $\widehat{A}_{2}(\cdot)$ & $\widehat{A}_{3}(\cdot)$ & $\widehat{A}_{4}(\cdot)$ & $\widehat{A}_{5}(\cdot)$ & $\widehat{A}_{6}(\cdot)$ \\
\hline C1 & 3.6343 & -0.4814 & -0.0075 & -0.0258 & 0.0189 & 0.0193 \\
C2 & 3.4345 & -0.3923 & 0.0416 & 0.0265 & -0.0709 & -0.0572 \\
C3 & 3.2031 & -0.1364 & -0.0088 & 0.0221 & 0.0430 & 0.0289 \\
C4 & 3.1445 & -0.1118 & 0.0041 & 0.0337 & 0.0062 & 0.0072 \\
C5 & 3.1015 & -0.0693 & -0.0345 & 0.0352 & -0.0112 & 0.0003 \\
C6 & 3.1347 & -0.1397 & 0.0020 & 0.0300 & -0.0061 & -0.0002 \\
C7 & 4.0591 & -0.5487 & -0.0907 & 0.0951 & 0.0992 & 0.0842 \\
C8 & 3.8032 & -0.5500 & -0.1007 & 0.0633 & 0.0139 & 0.0277 \\
C9 & 4.5095 & -0.7435 & -0.1134 & 0.1809 & 0.2231 & 0.2026 \\
C10 & 3.6321 & -0.4685 & -0.0540 & 0.0384 & -0.0152 & 0.0011 \\
C11 & 3.2967 & -0.2274 & -0.0083 & 0.0553 & 0.0250 & 0.0240 \\
C12 & 3.3454 & -0.2122 & -0.0927 & -0.0330 & 0.0724 & 0.0679 \\
C13 & 4.8419 & -0.6790 & -0.2455 & 0.0311 & 0.0554 & 0.0667 \\
C14 & 3.0941 & -0.1037 & 0.0210 & 0.0141 & -0.0016 & 0.0041 \\
C15 & 3.2877 & -0.2598 & -0.0524 & 0.0842 & -0.0423 & -0.0348 \\
C16 & 3.6870 & -0.4302 & -0.0086 & 0.0078 & -0.0027 & -0.0017 \\
C17 & 3.2197 & -0.2071 & 0.0162 & 0.0079 & 0.0206 & 0.0110 \\
\hline
\end{tabular}

Bootstrap curve confidence intervals at confidence level $1-\alpha=0.95$, based on 1000 bootstrap samples, are computed for the spatial mean, over the 17 Spanish Communities, of the curve regression predictors. Their construction is based on the bias corrected and accelerated percentile method $\left(\mathcal{I}_{1}\right)$; Normal approximated interval with bootstrapped bias and standard error $\left(\mathcal{I}_{2}\right)$; basic 
Table 2: Regression parameter estimates $\widehat{B}_{k}(\cdot), k=1, \ldots, 6$, at the 17 Spanish Communities

\begin{tabular}{|ccccccc|}
\hline SC $/$ PE & $\widehat{B}_{1}(\cdot)$ & $\widehat{B}_{2}(\cdot)$ & $\widehat{B}_{3}(\cdot)$ & $\widehat{B}_{4}(\cdot)$ & $\widehat{B}_{5}(\cdot)$ & $\widehat{B}_{6}(\cdot)$ \\
\hline C1 & 0 & -0.0052 & -0.1330 & -0.0123 & 0.0064 & -0.0195 \\
C2 & 0 & -0.0367 & -0.0998 & -0.0462 & -0.0343 & -0.0107 \\
C3 & 0 & -0.0531 & -0.0074 & -0.0142 & -0.0003 & 0.0020 \\
C4 & 0 & -0.0074 & -0.0284 & -0.0151 & -0.0092 & 0.0012 \\
C5 & 0 & 0.0433 & -0.0438 & -0.0116 & -0.0118 & 0.0046 \\
C6 & 0 & 0.0018 & -0.0174 & -0.0068 & -0.0089 & 0.0000 \\
C7 & 0 & -0.0365 & -0.2451 & -0.1791 & -0.0820 & 0.0026 \\
C8 & 0 & 0.0953 & -0.2389 & -0.0431 & -0.0313 & -0.0045 \\
C9 & 0 & -0.1587 & -0.4054 & -0.2269 & -0.1010 & 0.0047 \\
C10 & 0 & 0.1118 & -0.1579 & -0.0458 & -0.0418 & -0.0220 \\
C11 & 0 & 0.0754 & -0.1138 & -0.0166 & -0.0048 & 0.0072 \\
C12 & 0 & -0.1104 & -0.1338 & 0.1330 & 0.0761 & -0.0017 \\
C13 & 0 & 0.4654 & -0.1302 & -0.1602 & -0.1061 & -0.0038 \\
C14 & 0 & 0.0355 & -0.0560 & 0.0119 & 0.0025 & -0.0044 \\
C15 & 0 & -0.0187 & -0.0021 & -0.0897 & -0.0562 & 0.0134 \\
C16 & 0 & 0.0025 & -0.0707 & -0.0638 & -0.0439 & -0.0267 \\
C17 & 0 & 0.0389 & -0.0270 & -0.0174 & -0.0006 & 0.0019 \\
\hline
\end{tabular}

percentile method $\left(\mathcal{I}_{3}\right)$, and bias corrected percentile method $\left(\mathcal{I}_{4}\right)$ (see Figure 1 below). The minimized regression empirical risk values $L_{265}\left(\widehat{\boldsymbol{\theta}}_{265}(p)\right), p=$ $1, \ldots, 17$, are displayed in Table 3 .

\begin{tabular}{ccccc} 
Table 3: Observed values & \multicolumn{4}{c}{$L_{265}\left(\widehat{\boldsymbol{\theta}}_{265}(p)\right), p=1, \ldots, 17$} \\
\hline$L_{265}\left(\widehat{\boldsymbol{\theta}}_{265}(p)\right)$ & $p=$ & $1 \ldots 17$ & & \\
\hline 0.0155 & 0.0259 & 0.0668 & 0.0408 & 0.0927 \\
0.0623 & 0.1642 & 0.0883 & 0.2174 & 0.0313 \\
0.0559 & 0.1904 & 0.0054 & 0.1602 & 0.1640 \\
0.0003 & 0.1238 & & & \\
\hline
\end{tabular}

Figure 2 at the top displays the 1000 bootstrap sample values

$$
\overline{L_{265}}\left(\omega_{i}\right)=\frac{1}{P} \sum_{p=1}^{P} L_{265}\left(\omega_{i}, \widehat{\boldsymbol{\theta}}_{265}(p)\right), \quad \omega_{i} \in \Omega, i=1, \ldots, 1000,
$$

of the spatial averaged minimized empirical quadratic risk in the trigonometric regression. Note that the sample mean of these values is $\overline{\bar{L}}=0.0262$, showing a good performance of the least-squares regression predictor, according to the value $T(265,12)=1.1304$ obtained. The bootstrap histogram and the corresponding approximation of the probability density function, computed from $\overline{L_{265}}\left(\omega_{i}\right), i=1, \ldots, 1000$, are also plotted at the bottom of Figure 2 . 

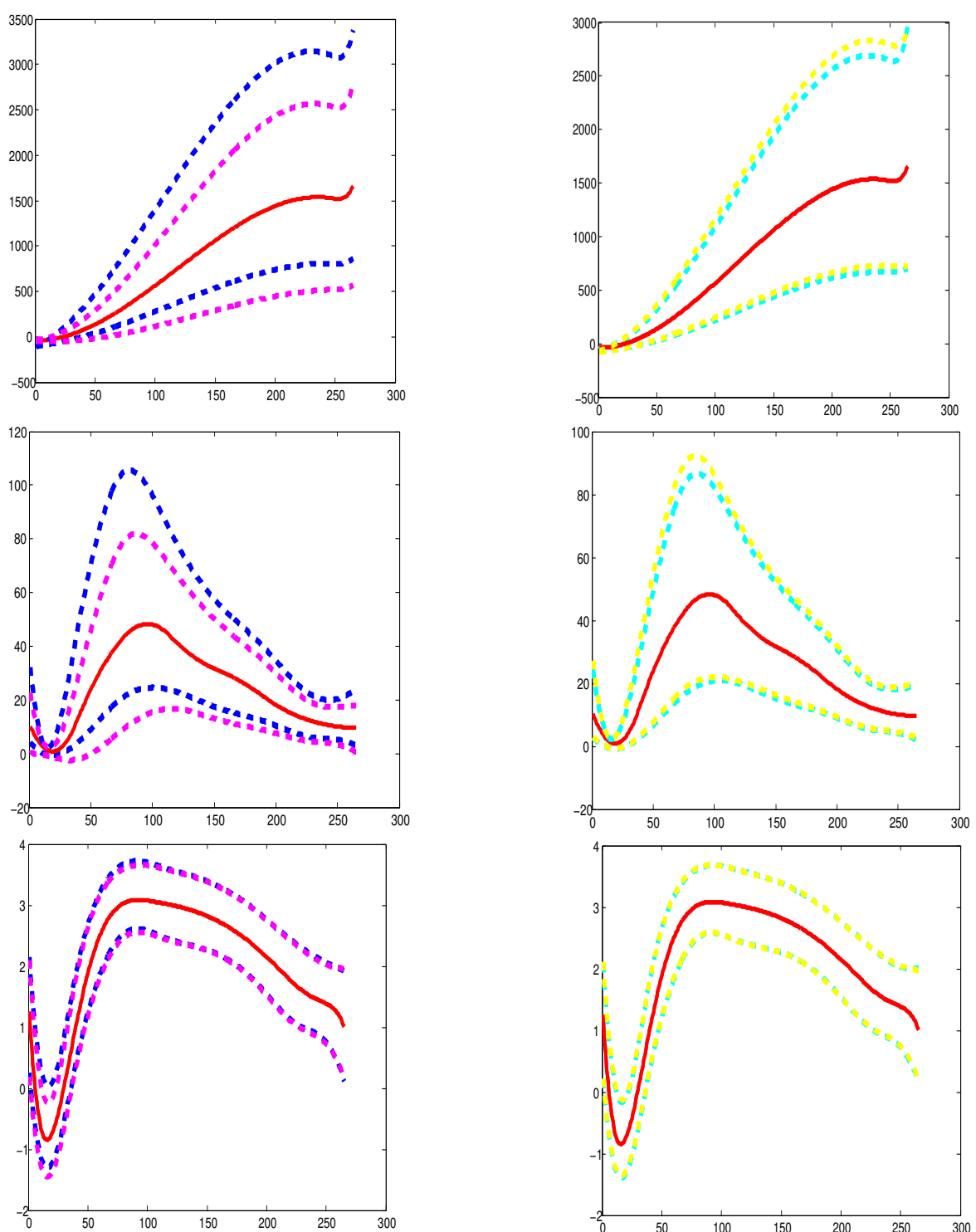

Fig. 1: At the top, COVID-19 mortality mean cumulative curve in Spain, since March, 8, 2020 to May, 13, 2020 (continuous red line, 265 temporal nodes), and bootstrap curve confidence intervals, at the left-hand-side, $\mathcal{I}_{1}$ (dashed blue lines) and $\mathcal{I}_{2}$ (dashed magenta lines), and at the right-hand-side, $\mathcal{I}_{3}$ (dashed green lines) and $\mathcal{I}_{4}$ (dashed yellow lines). Plots at the center and bottom reflect the same information respectively referred to the mean intensity (spatial averaged COVID-19 mortality risk curve), and log-intensity (spatial averaged COVID-19 mortality log-risk curve) curves in Spain. All the confidence bootstrap intervals are computed at confidence level $1-\alpha=0.95$, from 1000 bootstrap samples 


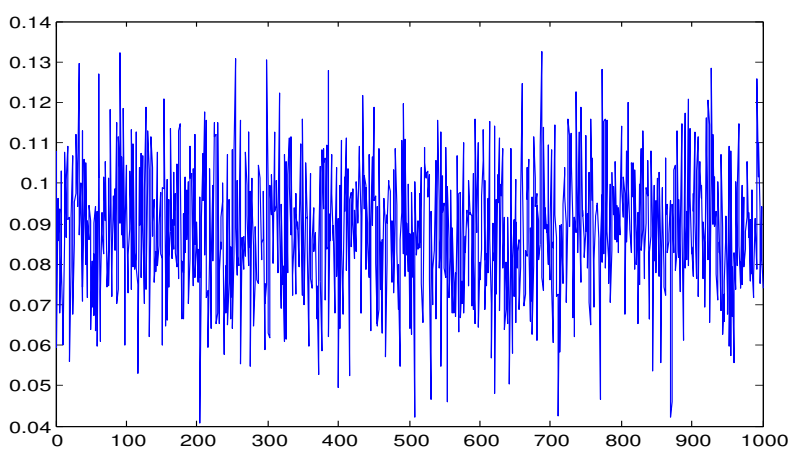

BOOTSTRAP HISTOGRAM

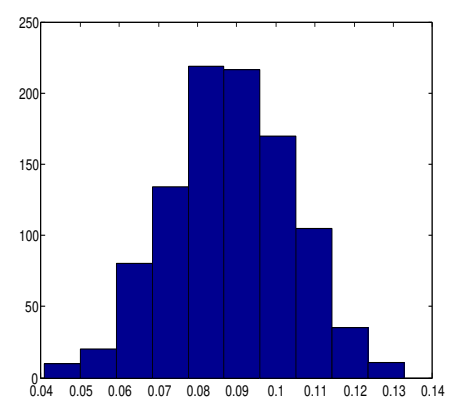

BOOTSTRAP PROBABILITY DENSITY

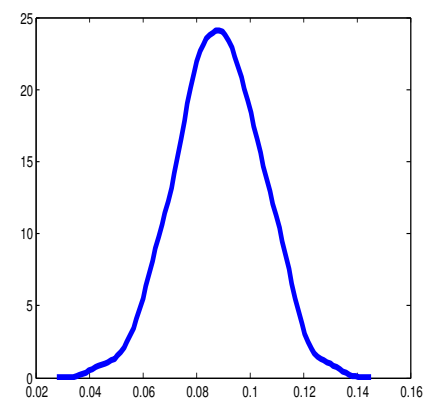

Fig. 2: At the top, sample values of the spatial mean of the minimized empirical risk in the trigonometric regression. Bootstrap histogram (left-hand side-bottom), and bootstrap probability density (right-hand-side-bottom) of the spatial mean of the minimized regression empirical risk, based on 1000 bootstrap samples

Bootstrap confidence intervals for $\overline{L_{265}}$ have also been computed at level $1-\alpha=0.95$, from 1000 and 10000 bootstrap samples. Table 4 displays these intervals respectively based on the bias corrected and accelerated percentile method $\left(\mathcal{I}_{1}\right)$; Normal approximated interval with bootstrapped bias and standard error $\left(\mathcal{I}_{2}\right)$; basic percentile method $\left(\mathcal{I}_{3}\right)$; bias corrected percentile method $\left(\mathcal{I}_{4}\right)$, and Student-based confidence interval $\left(\mathcal{I}_{5}\right)$.

The classical and Bayesian plug-in predictors of the residual COVID-19 mortality log-risk process at each one of the Spanish Communities are respectively computed from equations (14) and (16) for $P=17$.

Given the empirical spectral characteristics observed in the regularized approximation $\widehat{\rho}_{k(T)}$ of $\rho$ in (12), from the singular value decomposition of the empirical operators in (11), our choice of the prior for the projections of $\rho$ has been a scaled, by factor $1 / 3$, Beta prior with hyper-parameters $a_{p q}=14$, and $b_{p q}=13$, for $p, q=1, \ldots, 17$. The suitability of this data-driven choice, regarding localization of the mode, and the tails thickness, is illustrated in 
Table 4: Bootstrap confidence intervals for $\overline{L_{265}}$ (confidence level $1-\alpha=0.95$ )

\begin{tabular}{ccc}
\hline $\mathrm{CI} / \mathrm{S}$ & 1000 & 10000 \\
$\mathcal{I}_{1}$ & {$[0.0593,0.1222]$} & {$[0.0594,0.1236]$} \\
$\mathcal{I}_{2}$ & {$[0.0564,0.1196]$} & {$[0.0567,0.1207]$} \\
$\mathcal{I}_{3}$ & {$[0.0584,0.1215]$} & {$[0.0579,0.1217]$} \\
$\mathcal{I}_{4}$ & {$[0.0592,0.1233]$} & {$[0.0581,0.1208]$} \\
$\mathcal{I}_{5}$ & {$[0.0484,0.1281]$} & {$[0.0494,0.1215]$} \\
\hline
\end{tabular}

Figure 3. Specifically, at the right plot in Figure 3, both, the scaled Beta probability density, with shape parameters 14 and 13 (red-square line), and the fitted probability density (blue-square line), from the generated bootstrap samples, based on the empirical projections of $\rho$, are displayed. Note that the observed range of the empirical projections of $\rho$ is well fitted, as one can see from the left plot in Figure 3.

17X17=289 EMPIRICAL PROJECTIONS OF RHO

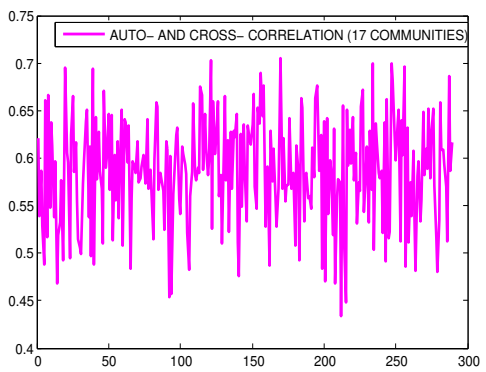

THEORETICAL AND EMPIRICAL PRIOR

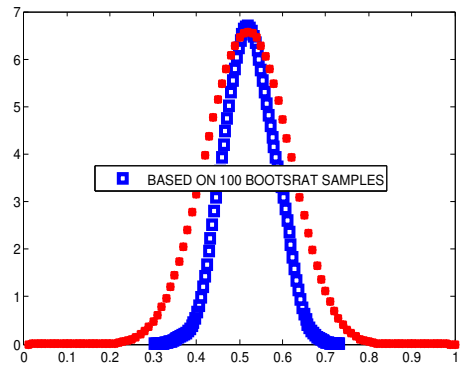

Fig. 3: At the left-hand side, empirical projections of the autocorrelation operator $\rho$, reflecting temporal autocorrelation and cross-correlation between the 17 Spanish Communities analyzed. At the right-hand side, the considered prior probability density (red squares) of a scaled, by factor $1 / 3$, Beta distributed random variable with shape parameters 14 and 13 is compared with the bootstrap fitting of an empirical prior (blue squares)

Bootstrap confidence intervals $\mathcal{I}_{1}, \ldots, \mathcal{I}_{5}$ at level $1-\alpha=0.95$, for the expected training standard error of the multivariate time series classical and 
Bayesian residual COVID-19 mortality log-risk predictors, based on 1000 bootstrap samples, are displayed in Table 5:

Table 5: Bootstrap confidence intervals for the expected training standard error of the classical and Bayesian residual COVID-19 mortality log-risk predictors $(1-\alpha=0.95)$

\begin{tabular}{ccc}
\hline CI/S & Classical & Bayesian \\
$\mathcal{I}_{1}$ & {$[0.0474,0.0597]$} & {$[0.0173,0.0228]$} \\
$\mathcal{I}_{2}$ & {$[0.0455,0.0578]$} & {$[0.0167,0.0220]$} \\
$\mathcal{I}_{3}$ & {$[0.0463,0.0588]$} & {$[0.0169,0.0225]$} \\
$\mathcal{I}_{4}$ & {$[0.0460,0.0586]$} & {$[0.0172,0.0226]$} \\
$\mathcal{I}_{5}$ & {$[0.0421,0.0563]$} & {$[0.0158,0.0215]$} \\
\hline
\end{tabular}

Maps plotted in Figure 5 show the observed spatiotemporal evolution of COVID-19 mortality risk, and its prediction, from the fitted curve trigonometric regression model, and the subsequent classical and Bayesian time series analysis.
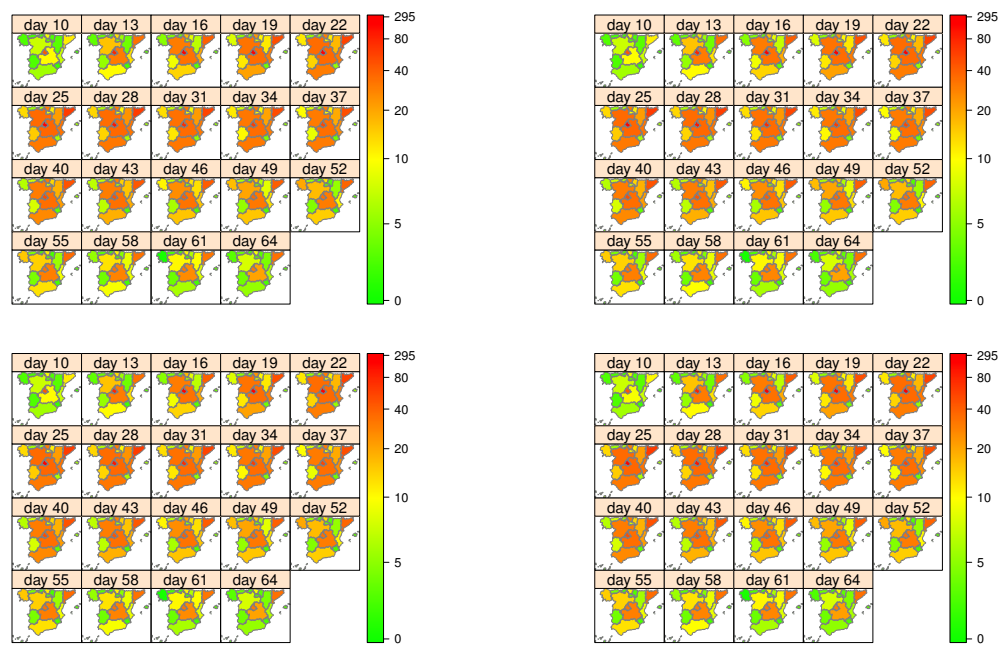

Fig. 4: COVID-19 mortality risk maps, since March, 8 to May, 13, 2020. Observed (lefthand-side) and estimated (right-hand side) maps, computed from trigonometric regression, combined with classical (first line) and Bayesian (second line) residual predictors 


\section{An empirical comparative study}

The ML regression models introduced in the Supplementary Material are applied to COVID-19 mortality analysis, and compared, via random $k$-fold cross-validation and bootstrap estimators, with the multiple objective spacetime forecasting approach presented. We distinguish two categories respectively referred to the strong-sense (hard-data) and weak-sense (soft-data) definition of our data set. Random $k$-fold $(k=5,10)$ cross-validation, in terms of Symmetric Mean Absolute Percentage Errors (SMAPEs), evaluates the performance of the compared regression models, from hard- and soft-data. Bootstrap confidence intervals, and probability density estimates of the spatially averaged SMAPEs are also computed. Section 6 provides a data-driven model classification, based on SMAPEs, in the two categories analyzed, from random $k$-fold cross-validation, and the bootstrap estimation procedures applied.

\subsection{Results from random $k$-fold cross-validation}

After interpolation and cubic $B$-spline smoothing of our original data set, the logarithmic transform and linear scaling are applied. We held out the first ten points and the last three, for each COVID-19 mortality log-risk curve, as an out of sample set. Our approach is implemented in the second-category from soft-data. In this implementation, we consider $N=6$, adopting the model selection criterion given in Section 4 (see equation (8) and reference [14]). In the multivariate time series classical and Bayesian prediction, our choice of $k(T)=k(265)=8$ provides a balance between $k(T)=[\ln (T)]^{-}=$ $[\ln (265)]^{-}=5$, signing an agreement with the separation and velocity decay of the empirical eigenvalues of the autocovariance operator, and the parameter value $k(T)=9$, controlling model complexity according to the sample size $T=265$. The random fluctuations, observed in the empirical projections of the spatial autocorrelation operator $\rho$ for this $k(T)$ parameter value, are also well-fitted by our previous choice of the shape hyperparameters, in the prior Beta probability density.

Model fitting is evaluated in terms of the Symmetric Mean Absolute Percentage Errors (SMAPEs), given by, for $P=17$, and $T=265$,

$$
\frac{1}{T} \sum_{t=1}^{T} \frac{\left|\widehat{\ln \left(\Lambda_{t}\right)}\left(\psi_{p, \rho_{p}}\right)-\ln \left(\Lambda_{t}\right)\left(\psi_{p, \rho_{p}}\right)\right|}{\left(\left|\ln \left(\Lambda_{t}\right)\left(\psi_{p, \rho_{p}}\right)\right|+\left|\widehat{\ln \left(\Lambda_{t}\right)}\left(\psi_{p, \rho_{p}}\right)\right|\right) / 2}, \quad p=1, \ldots, P .
$$

We have computed the mean of the SMAPEs obtained at each one of the $k$ iterations of the random $k$-fold cross-validation procedure. This validation technique consists of random splitting the functional sample into a training and validation samples at each one of the $k$ iterations. Model fitting is performed from the training sample, and the target outputs are defined from the validation or testing sample. By running each model ten times and averaging 
SMAPEs, we remove the fluctuations due to the random initial weights (for MLP and BNN models), and the differences in the parameter estimation in all methods, due to the random specification of the sample splitting in the random $k$-fold cross-validation procedure.

The ten-running based random 10-fold cross-validation SMAPEs are displayed in Table 6, for the six ML techniques tested, GRNN, MLP, SVR, BNN, $\mathrm{RBF}$, and GP, when hard-data are considered (see also Table 3 of the Supplementary Material on random 5-fold cross-validation results). Table 7 provides the ten-running based random 10-fold cross-validation results, from soft-data category (see also Table 4 of the Supplementary Material on random 5-fold cross validation results). The corresponding cross-validation results of the presented approach from soft-data are displayed in Table 8.

ML model hyperparameter selection has been achieved by applying random $k$-fold cross-validation $(k=5,10)$. Our selection has been made from a suitable set of candidates. Specifically, the optimal numbers of hidden $(\mathrm{NH})$ nodes in the implementation of MLP and BNN have been selected from the candidate sets $[0,1,3,5,7,9]$ and $[1,3,5,7,9]$, respectively. The random crossvalidation results in both cases, $k=5,10$, lead to the same choice of the $\mathrm{NH}$ optimal value. Namely, $\mathrm{NH}=1$ for MLP, and $\mathrm{NH}=5$ for $\mathrm{BNN}$. The last one displays slight differences with respect to the values $\mathrm{NH}=3,7$, in the random 10 -fold cross-validation implementation. In the same way, we have selected the respective spread $\beta$ and bandwidth $h$ parameters in the RBF and GRNN procedures. Thus, after applying random $k$-fold cross-validation, with $k=5,10$, the optimal values $\beta=2.5$, and $h=0.05$ are obtained, from the candidate sets $[2.5,5,7.5,10,12.5,15,17.5,20]$ and $[0.05,0.1,0.2,0.3,0.5,0.6,0.7]$, respectively (see Supplementary Material). Better performance from hard-data is observed in linear SVR. In its implementation, automatic hyperparameter optimization from fitrsvm MatLab function is applied. While, from the soft-data category, the best option corresponds to the Gaussian kernel based nonlinear SVR model fitting (applying the same option of automatic hyperparameter optimization, in the argument of fitrsvm MatLab function). In the implementation of GP, we follow the same tuning procedure for model selection. In this case, for both categories, we have selected Bayesian cross-validation optimization (in the hyperparameter optimization argument of the fitrgp MatLab function).

In all the results displayed, the SMAPE-MEAN (M.) and SMAPE-TOTAL (T.) have been computed as performance measures, for comparing the ML models tested, and our approach.

\subsection{Bootstrap based classification results}

For the ML regression models tested, in the hard- and soft-data categories, bootstrap confidence intervals $(1-\alpha=0.95$ confidence level) for the spatially averaged SMAPEs, based on 1000 bootstrap samples, are constructed. Our approach requires the soft-data information to be incorporated. As before, the computed bootstrap confidence intervals $\mathcal{I}_{i}, i=1, \ldots, 5$, are respectively 
Table 6: Hard-data category. Averaged SMAPEs, based on 10 running of random 10-fold cross-validation

\begin{tabular}{|c|c|c|c|c|c|c|}
\hline $\mathrm{SC}\left(\mathrm{x} 10^{-2}\right)$ & GRNN & MLP & SVR & BNN & RBF & GP \\
\hline C1 & 0.1957 & 0.0777 & 0.0700 & 0.0594 & 0.0543 & 0.0554 \\
\hline $\mathrm{C} 2$ & 0.6132 & 0.1490 & 0.0663 & 0.0738 & 0.0680 & 0.0654 \\
\hline $\mathrm{C} 3$ & 0.1556 & 0.0473 & 0.0350 & 0.0303 & 0.0331 & 0.0304 \\
\hline $\mathrm{C} 4$ & 0.0971 & 0.0342 & 0.0135 & 0.0200 & 0.0182 & 0.0211 \\
\hline C5 & 0.2049 & 0.0457 & 0.0318 & 0.0370 & 0.0369 & 0.0372 \\
\hline $\mathrm{C} 6$ & 0.1572 & 0.0368 & 0.0177 & 0.0234 & 0.0233 & 0.0247 \\
\hline $\mathrm{C} 7$ & 0.4898 & 0.0698 & 0.0644 & 0.0590 & 0.0616 & 0.0588 \\
\hline C8 & 0.0804 & 0.0340 & 0.0171 & 0.0191 & 0.0211 & 0.0177 \\
\hline C9 & 0.7258 & 0.1976 & 0.0979 & 0.0812 & 0.0326 & 0.0437 \\
\hline C10 & 0.2191 & 0.0704 & 0.0556 & 0.0482 & 0.0471 & 0.0463 \\
\hline C11 & 0.1262 & 0.0530 & 0.0310 & 0.0395 & 0.0375 & 0.0355 \\
\hline C12 & 0.5228 & 0.1578 & 0.1341 & 0.1282 & 0.0940 & 0.0993 \\
\hline $\mathrm{C} 13$ & 0.3594 & 0.0647 & 0.0576 & 0.0579 & 0.0533 & 0.0458 \\
\hline C14 & 0.1345 & 0.0366 & 0.0209 & 0.0204 & 0.0194 & 0.0207 \\
\hline C15 & 0.6080 & 0.1523 & 0.1411 & 0.1141 & 0.0982 & 0.1039 \\
\hline C16 & 0.2464 & 0.0889 & 0.0709 & 0.0622 & 0.0568 & 0.0594 \\
\hline $\mathrm{C} 17$ & 0.0660 & 0.0370 & 0.0148 & 0.0222 & 0.0203 & 0.0227 \\
\hline M. & 0.2942 & 0.0796 & 0.0553 & 0.0527 & 0.0456 & 0.0463 \\
\hline T. & 5.0022 & 1.3528 & 0.9397 & 0.8959 & 0.7757 & 0.7879 \\
\hline
\end{tabular}

Table 7: Soft-data category. Averaged SMAPEs, based on 10 running of random 10-fold cross-validation

\begin{tabular}{|c|c|c|c|c|c|c|}
\hline SC $\left(\mathbf{x 1 0}^{-\mathbf{2}}\right)$ & GRNN & MLP & SVR & BNN & RBF & GP \\
\hline C1 & 0.1545 & 0.0983 & 0.0666 & 0.0573 & 0.0234 & 0.0312 \\
C2 & 0.1844 & 0.1730 & 0.0660 & 0.0749 & 0.0277 & 0.0301 \\
C3 & 0.1029 & 0.1192 & 0.0481 & 0.0452 & 0.0273 & 0.0274 \\
C4 & 0.0432 & 0.0286 & 0.0165 & 0.0158 & 0.0124 & 0.0123 \\
C5 & 0.0610 & 0.0476 & 0.0258 & 0.0248 & 0.0144 & 0.0149 \\
C6 & 0.0260 & 0.0217 & 0.0133 & 0.0140 & 0.0124 & 0.0125 \\
C7 & 0.3750 & 0.2026 & 0.1095 & 0.0924 & 0.0307 & 0.0399 \\
C8 & 0.0764 & 0.0482 & 0.0305 & 0.0300 & 0.0262 & 0.0187 \\
C9 & 0.4894 & 0.3198 & 0.1753 & 0.1212 & 0.0229 & 0.0372 \\
C10 & 0.1680 & 0.0815 & 0.0521 & 0.0462 & 0.0252 & 0.0290 \\
C11 & 0.1537 & 0.0839 & 0.0436 & 0.0397 & 0.0199 & 0.0219 \\
C12 & 0.3689 & 0.2558 & 0.1505 & 0.1249 & 0.0401 & 0.0490 \\
C13 & 0.2848 & 0.1582 & 0.0968 & 0.0792 & 0.0240 & 0.0320 \\
C14 & 0.0367 & 0.0226 & 0.0120 & 0.0143 & 0.0106 & 0.0104 \\
C15 & 0.3618 & 0.2264 & 0.1201 & 0.1227 & 0.0317 & 0.0522 \\
C16 & 0.1773 & 0.0835 & 0.0651 & 0.0545 & 0.0264 & 0.0318 \\
C17 & 0.0884 & 0.0623 & 0.0210 & 0.0231 & 0.0125 & 0.0136 \\
\hline \hline M. & 0.1854 & 0.1196 & 0.0655 & 0.0577 & 0.0228 & 0.0273 \\
\hline T. & 3.1524 & 2.0333 & 1.1129 & 0.9801 & 0.3877 & 0.4642 \\
\hline
\end{tabular}

based on the bias corrected and accelerated percentile method $\left(\mathcal{I}_{1}\right)$; Normal approximated interval with bootstrapped bias and standard error $\left(\mathcal{I}_{2}\right)$; basic percentile method $\left(\mathcal{I}_{3}\right)$; bias corrected percentile method $\left(\mathcal{I}_{4}\right)$, and Studentbased confidence interval $\left(\mathcal{I}_{5}\right)$ (see Tables 9 and 10). The bootstrap histogram, 
Table 8: Our approach. Averaged SMAPEs, based on 10 running of random 10-fold crossvalidation of the tested Classical (C.) and Bayesian (B.) residual analysis

\begin{tabular}{|c|c|c|}
\hline SC & C. k10 & B. k10 \\
\hline & & \\
C1 & 0.0024 & $0.7106(10)^{-3}$ \\
C2 & 0.0019 & $0.4003(10)^{-3}$ \\
C3 & 0.0016 & $0.6797(10)^{-3}$ \\
C4 & 0.0017 & $0.4367(10)^{-3}$ \\
C5 & 0.0023 & $0.6530(10)^{-3}$ \\
C6 & 0.0018 & $0.5854(10)^{-3}$ \\
C7 & 0.0017 & $0.6341(10)^{-3}$ \\
C8 & 0.0016 & $0.6593(10)^{-3}$ \\
C9 & 0.0013 & $0.5979(10)^{-3}$ \\
C10 & 0.0019 & $0.6954(10)^{-3}$ \\
C11 & 0.0017 & $0.5444(10)^{-3}$ \\
C12 & 0.0016 & $0.5016(10)^{-3}$ \\
C13 & 0.0020 & $0.4832(10)^{-3}$ \\
C14 & 0.0026 & $0.6544(10)^{-3}$ \\
C15 & 0.0023 & $0.6616(10)^{-3}$ \\
C16 & 0.0015 & $0.7134(10)^{-3}$ \\
C17 & 0.0022 & $0.6781(10)^{-3}$ \\
\hline \hline M. & 0.0019 & $0.60524(10)^{-3}$ \\
\hline T. & 0.0321 & 0.0103 \\
\hline
\end{tabular}

and probability density of the spatially averaged SMAPEs are displayed in Figures 5 and 6, for the hard-data category, and in Figures 7, 8, and 9, for the soft-data category. The data-driven performance-based model classification results obtained are discussed in Section 6 . 
Table 9: Hard-data category. Bootstrap confidence intervals $(1-\alpha=0.95)$ for the spatially averaged SMAPEs from 1000 bootstrap samples $(T=265, P=17)$

\begin{tabular}{lll}
\hline CI/ML & GRNN & MLP \\
$\mathcal{I}_{1}$ & {$\left[2.1(10)^{-3}, 4.1(10)^{-3}\right]$} & {$\left[0.5(10)^{-3}, 1(10)^{-3}\right]$} \\
$\mathcal{I}_{2}$ & {$\left[2(10)^{-3}, 3.9(10)^{-3}\right]$} & {$\left[0.4776(10)^{-3}, 0.9483(10)^{-3}\right]$} \\
$\mathcal{I}_{3}$ & {$\left[2(10)^{-3}, 4(10)^{-3}\right]$} & {$\left[0.4746(10)^{-3}, 0.9713(10)^{-3}\right]$} \\
$\mathcal{I}_{4}$ & {$\left[2(10)^{-3}, 4(10)^{-3}\right]$} & {$\left[0.5118(10)^{-3}, 0.9878(10)^{-3}\right]$} \\
$\mathcal{I}_{5}$ & {$\left[1.7(10)^{-3}, 3.9(10)^{-3}\right]$} & {$\left[0.2780(10)^{-3}, 0.9244(10)^{-3}\right]$} \\
\hline CI/ML & $\mathbf{S V R}$ & $\mathbf{B N N}$ \\
$\mathcal{I}_{1}$ & {$\left[0.3682(10)^{-3}, 0.7219(10)^{-3}\right]$} & {$\left[0.3720(10)^{-3}, 0.6659(10)^{-3}\right]$} \\
$\mathcal{I}_{2}$ & {$\left[0.3516(10)^{-3}, 0.6763(10)^{-3}\right]$} & {$\left[0.3587(10)^{-3}, 0.6379(10)^{-3}\right]$} \\
$\mathcal{I}_{3}$ & {$\left[0.3493(10)^{-3}, 0.6770(10)^{-3}\right]$} & {$\left[0.3668(10)^{-3}, 0.6509(10)^{-3}\right]$} \\
$\mathcal{I}_{4}$ & {$\left[0.3508(10)^{-3}, 0.6865(10)^{-3}\right]$} & {$\left[0.3654(10)^{-3}, 0.6379(10)^{-3}\right]$} \\
$\mathcal{I}_{5}$ & {$\left[0.3050(10)^{-3}, 0.6661(10)^{-3}\right]$} & {$\left[0.3099(10)^{-3}, 0.6335(10)^{-3}\right]$} \\
\hline $\mathrm{CI}_{\mathrm{ML}}$ & $\mathbf{R B F}$ & $\mathbf{G P}$ \\
$\mathcal{I}_{1}$ & {$\left[0.3260(10)^{-3}, 0.5310(10)^{-3}\right]$} & {$\left[0.3243(10)^{-3}, 0.5350(10)^{-3}\right]$} \\
$\mathcal{I}_{2}$ & {$\left[0.3155(10)^{-3}, 0.5159(10)^{-3}\right]$} & {$\left[0.3065(10)^{-3}, 0.5126(10)^{-3}\right]$} \\
$\mathcal{I}_{3}$ & {$\left[0.3140(10)^{-3}, 0.5270(10)^{-3}\right]$} & {$\left[0.3095(10)^{-3}, 0.5188(10)^{-3}\right]$} \\
$\mathcal{I}_{4}$ & {$\left[0.3247(10)^{-3}, 0.5338(10)^{-3}\right]$} & {$\left[0.3152(10)^{-3}, 0.5222(10)^{-3}\right]$} \\
$\mathcal{I}_{5}$ & {$\left[0.2677(10)^{-3}, 0.5141(10)^{-3}\right]$} & {$\left[0.2505(10)^{-3}, 0.5046(10)^{-3}\right]$} \\
\hline
\end{tabular}


Table 10: Soft-data category. Bootstrap confidence intervals $(1-\alpha=0.95)$ for the spatially averaged SMAPEs from 1000 bootstrap samples $(T=265, P=17)$

\begin{tabular}{lll}
\hline CI/ML & GRNN & MLP \\
$\mathcal{I}_{1}$ & {$\left[1.3(10)^{-3}, 2.6(10)^{-3}\right]$} & {$\left[0.6(10)^{-3}, 1.3(10)^{-3}\right]$} \\
$\mathcal{I}_{2}$ & {$\left[1.3(10)^{-3}, 2.6(10)^{-3}\right]$} & {$\left[0.6(10)^{-3}, 1.3(10)^{-3}\right]$} \\
$\mathcal{I}_{3}$ & {$\left[1.3(10)^{-3}, 2.7(10)^{-3}\right]$} & {$\left[0.6(10)^{-3}, 1.3(10)^{-3}\right]$} \\
$\mathcal{I}_{4}$ & {$\left[1.3(10)^{-3}, 2.6(10)^{-3}\right]$} & {$\left[0.7(10)^{-3}, 1.3(10)^{-3}\right]$} \\
$\mathcal{I}_{5}$ & {$\left[1(10)^{-3}, 2.7(10)^{-3}\right]$} & {$\left[0.5(10)^{-3}, 1.3(10)^{-3}\right]$} \\
\hline CI/ML & $\mathbf{S V R}$ & $\mathbf{B N N}$ \\
$\mathcal{I}_{1}$ & {$\left[0.4096(10)^{-3}, 0.8221(10)^{-3}\right]$} & {$\left[0.3588(10)^{-3}, 0.6177(10)^{-3}\right]$} \\
$\mathcal{I}_{2}$ & {$\left[0.3764(10)^{-3}, 0.7763(10)^{-3}\right]$} & {$\left[0.3433(10)^{-3}, 0.6053(10)^{-3}\right]$} \\
$\mathcal{I}_{3}$ & {$\left[0.3900(10)^{-3}, 0.7889(10)^{-3}\right]$} & {$\left[0.3454(10)^{-3}, 0.6037(10)^{-3}\right]$} \\
$\mathcal{I}_{4}$ & {$\left[0.4108(10)^{-3}, 0.7805(10)^{-3}\right]$} & {$\left[0.3559(10)^{-3}, 0.5988(10)^{-3}\right]$} \\
$\mathcal{I}_{5}$ & {$\left[0.3105(10)^{-3}, 0.7818(10)^{-3}\right]$} & {$\left[0.3003(10)^{-3}, 0.6129(10)^{-3}\right]$} \\
\hline $\mathrm{CI}_{\mathrm{ML}}$ & $\mathbf{R B F}$ & $\mathbf{G P}$ \\
$\mathcal{I}_{1}$ & {$\left[0.1794(10)^{-3}, 0.2478(10)^{-3}\right]$} & {$\left[0.2095(10)^{-3}, 0.3248(10)^{-3}\right]$} \\
$\mathcal{I}_{2}$ & {$\left[0.1754(10)^{-3}, 0.2474(10)^{-3}\right]$} & {$\left[0.2065(10)^{-3}, 0.3215(10)^{-3}\right]$} \\
$\mathcal{I}_{3}$ & {$\left[0.1785(10)^{-3}, 0.2485(10)^{-3}\right]$} & {$\left[0.2079(10)^{-3}, 0.3262(10)^{-3}\right]$} \\
$\mathcal{I}_{4}$ & {$\left[0.1743(10)^{-3}, 0.2494(10)^{-3}\right]$} & {$\left[0.2091(10)^{-3}, 0.3258(10)^{-3}\right]$} \\
$\mathcal{I}_{5}$ & {$\left[0.1616(10)^{-3}, 0.2542(10)^{-3}\right]$} & {$\left[0.1941(10)^{-3}, 0.3232(10)^{-3}\right]$} \\
\hline CI/OA & $\mathbf{C l a s s i c a l}$ & Bayesian \\
$\mathcal{I}_{1}$ & {$\left[2.2(10)^{-3}, 3.7(10)^{-3}\right]$} & {$\left[0.2943(10)^{-3}, 0.5177(10)^{-3}\right]$} \\
$\mathcal{I}_{2}$ & {$\left[2.1(10)^{-3}, 3.3(10)^{-3}\right]$} & {$\left[0.2802(10)^{-3}, 0.4854(10)^{-3}\right]$} \\
$\mathcal{I}_{3}$ & {$\left[2.1(10)^{-3}, 3.3(10)^{-3}\right]$} & {$\left[0.2833(10)^{-3}, 0.4884(10)^{-3}\right]$} \\
$\mathcal{I}_{4}$ & {$\left[2.2(10)^{-3}, 3.4(10)^{-3}\right]$} & {$\left[0.2900(10)^{-3}, 0.5124(10)^{-3}\right]$} \\
$\mathcal{I}_{5}$ & {$\left[1.8(10)^{-3}, 3.2(10)^{-3}\right]$} & {$\left[0.2418(10)^{-3}, 0.4664(10)^{-3}\right]$} \\
\hline & &
\end{tabular}




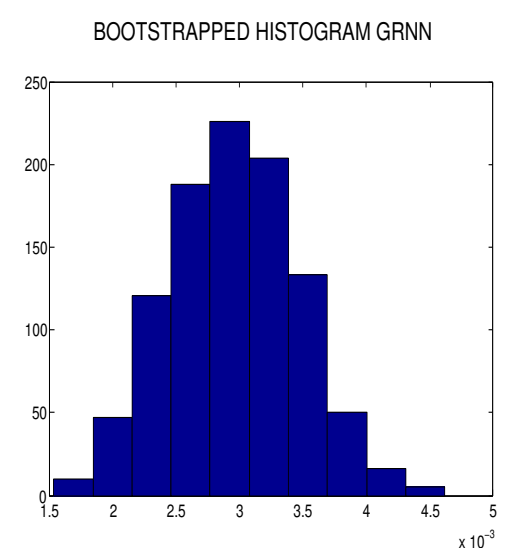

BOOTSTRAPPED HISTOGRAM MLP

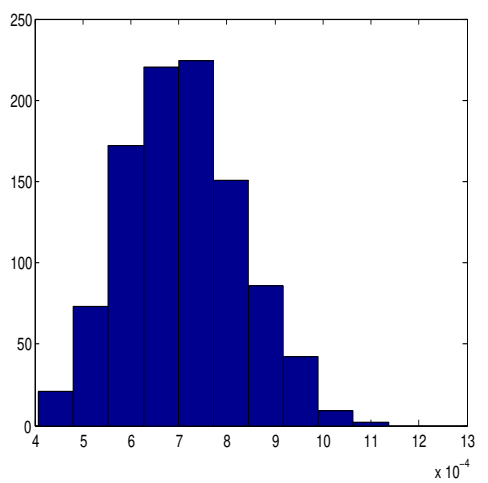

BOOTSTRAPPED HISTOGRAM SVR

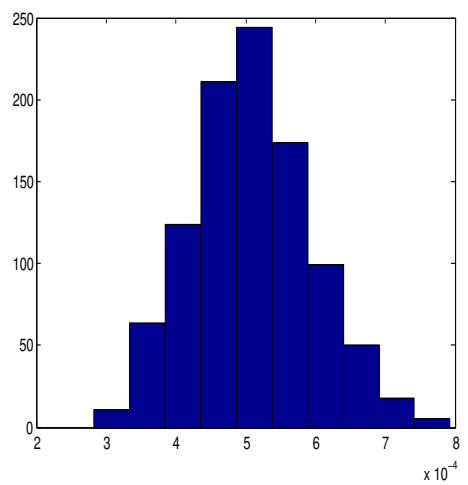

BOOTSTRAPPED PROBABILITY DENSITY GRNN

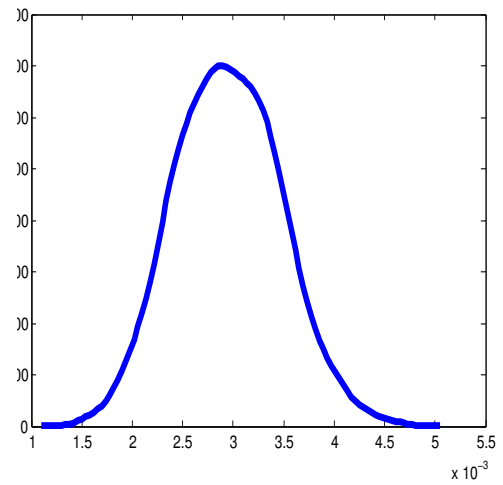

BOOTSTRAPPED PROBABILITY DENSITY MLP

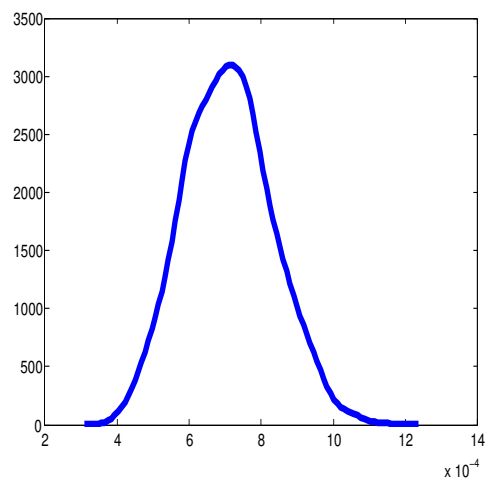

BOOTSTRAPPED PROBABILITY DENSITY SVR

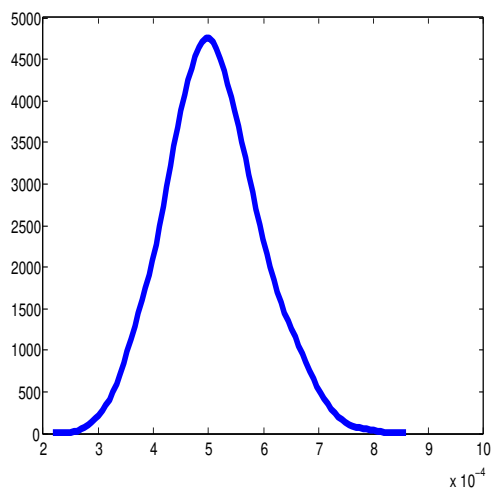

Fig. 5: Hard-data category. From 1000 bootstrap samples, spatially averaged SMAPEs histograms and probability densities are plotted, for GRNN (top), MLP (center), and linear SVR (bottom) 
BOOTSTRAPPED HISTOGRAM BNN

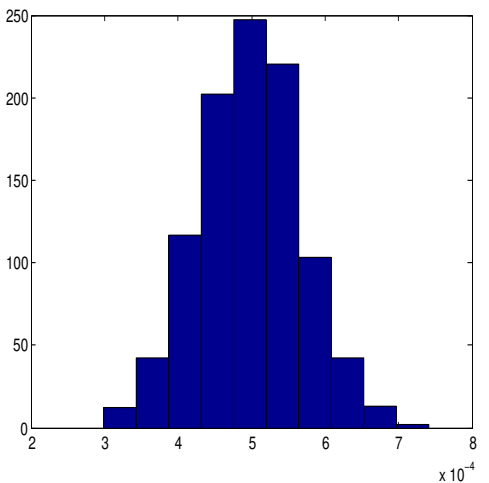

BOOTSTRAPPED HISTOGRAM RBF

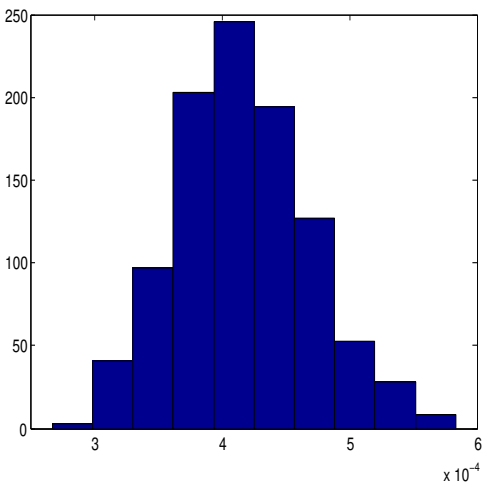

BOOTSTRAPPED HISTOGRAM GP

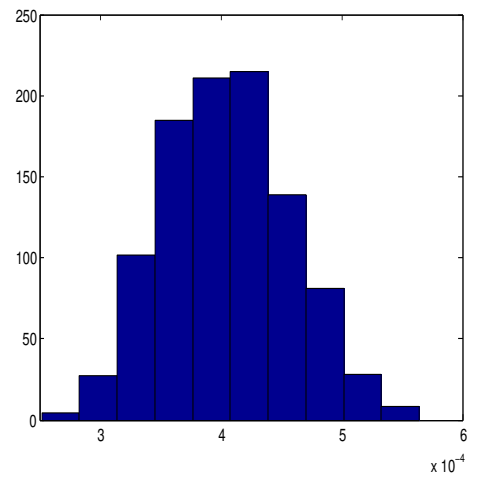

BOOTSTRAPPED PROBABILITY DENSITY BNN

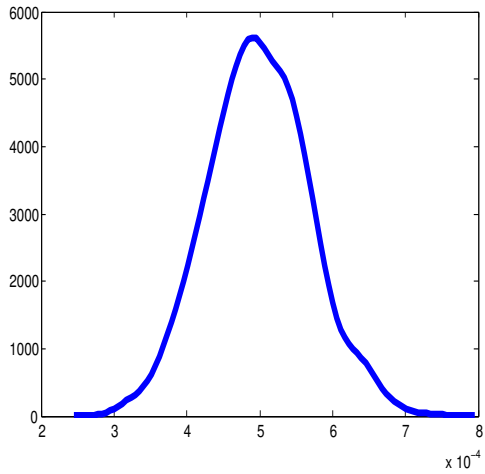

BOOTSTRAPPED PROBABILITY DENSITY RBF

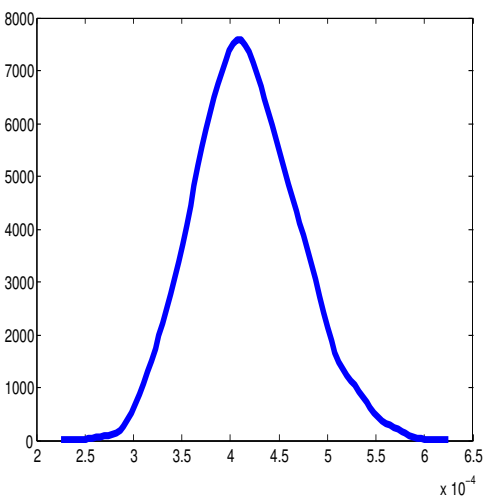

BOOTSTRAPPED PROBABILITY DENSITY GP

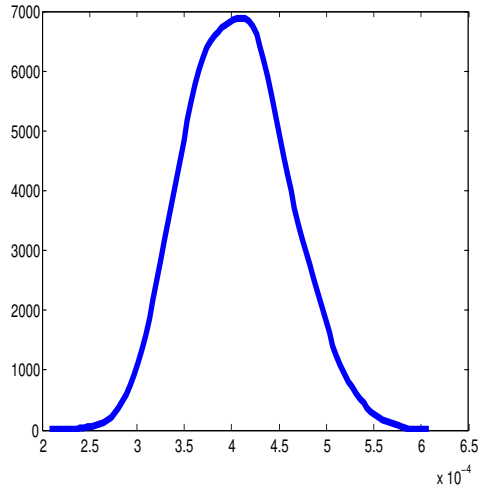

Fig. 6: Hard-data category. From 1000 bootstrap samples, spatially averaged SMAPEs histograms and probability densities are plotted, for BNN (top), RBF (center), and GP (bottom) 
BOOTSTRAPPED HISTOGRAM GRNN (SD)

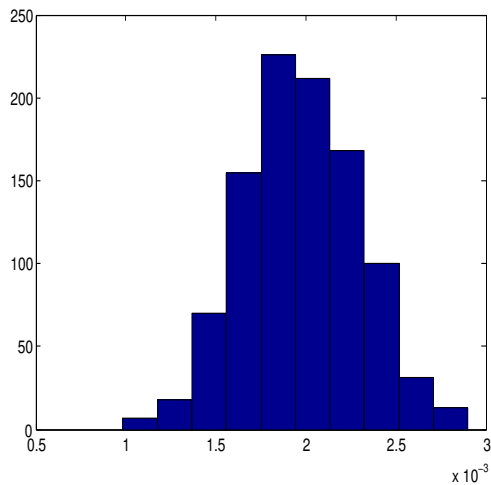

BOOTSTRAPPED HISTOGRAM MLP (SD)

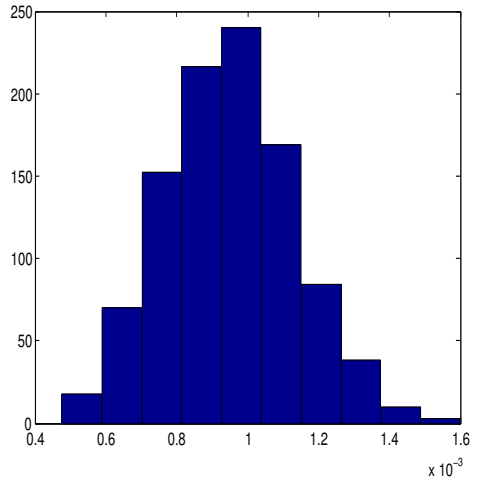

BOOTSTRAPPED HISTOGRAM SVR (SD)

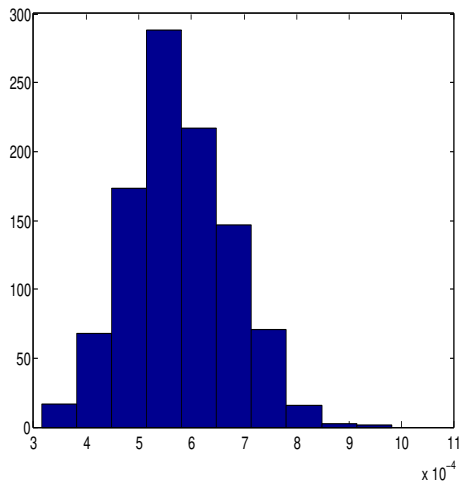

BOOTSTRAPPED PROBABILITY DENSITY GRNN (SD)

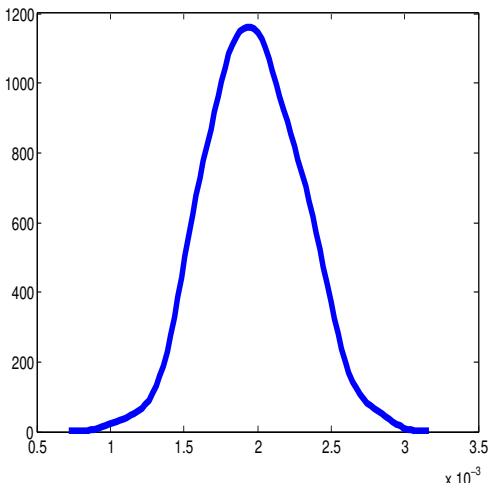

BOOTSTRAPPED PROBABILITY DENSITY MLP (SD)

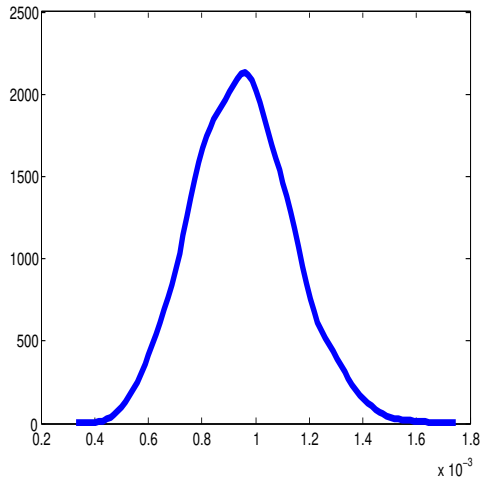

BOOTSTRAPPED PROBABILITY DENSITY SVR (SD)

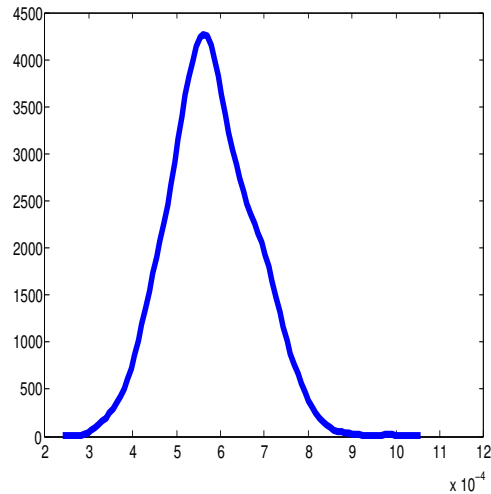

Fig. 7: Soft-data category. From 1000 bootstrap samples, spatially averaged SMAPEs histograms and probability densities are plotted, for GRNN (top), MLP (center) and nonlinear SVR (bottom) 
BOOTSTRAPPED HISTOGRAM BNN (SD)

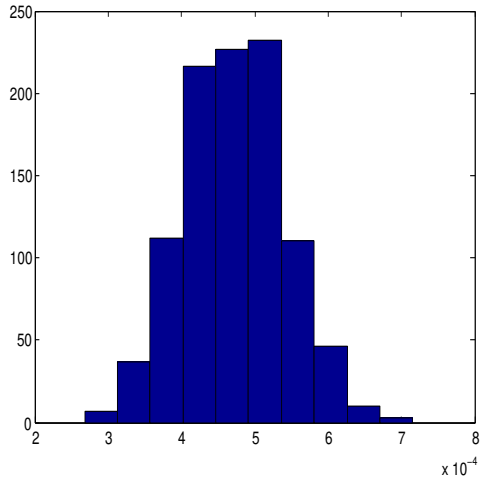

BOOTSTRAPPED HISTOGRAM RBF (SD)

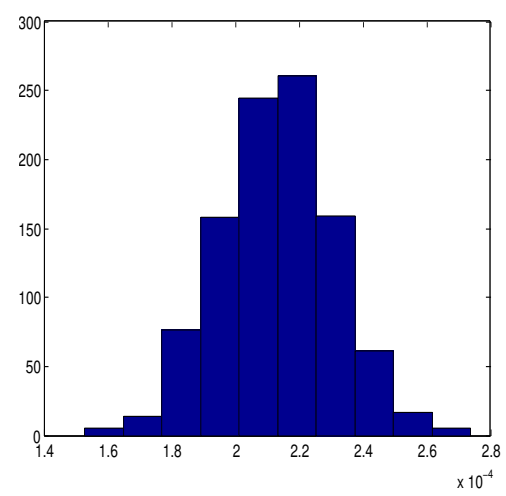

BOOTSTRAPPED HISTOGRAM GP (SD)

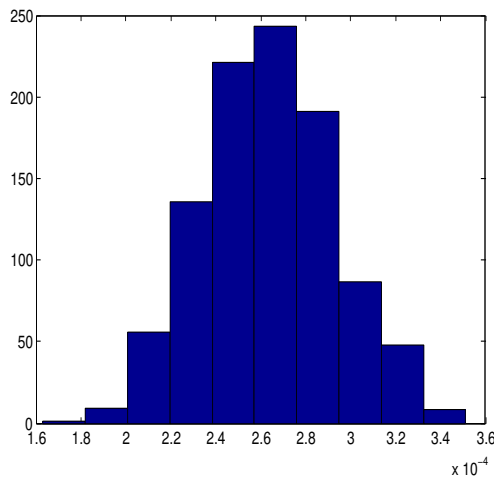

BOOTSTRAPPED PROBABILITY DENSITY BNN (SD)

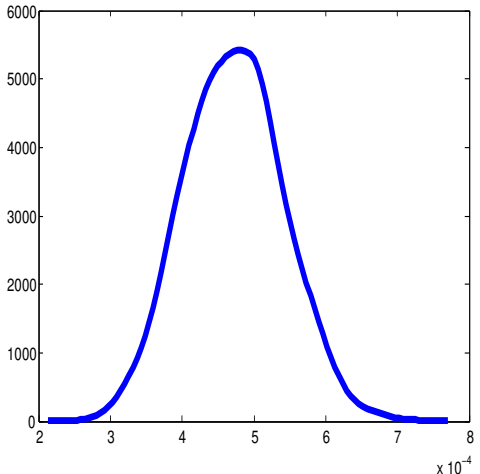

BOOTSTRAPPED PROBABILITY DENSITY RBF (SD)

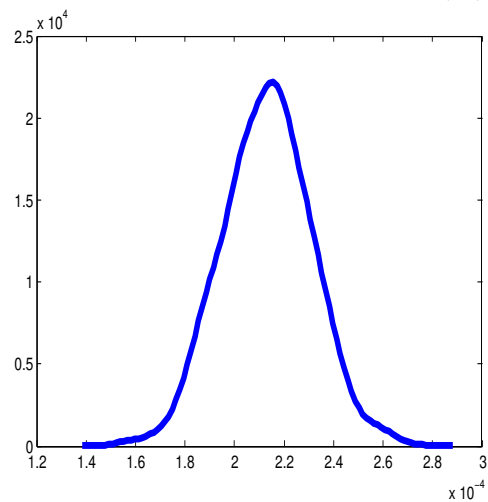

BOOTSTRAPPED PROBABILITY DENSITY GP (SD)

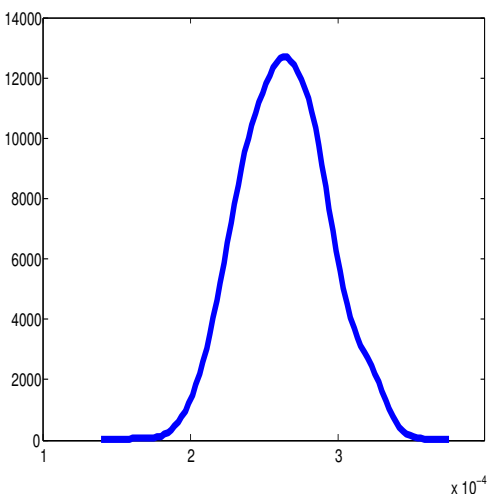

Fig. 8: Soft-data category. From 1000 bootstrap samples, spatially averaged SMAPEs histograms and probability densities are plotted, for BNN (top), RBF (center) and GP (bottom) 
CLASSICAL BOOTSTRAPPED HISTOGRAM (SD) CLASSICAL BOOTSTRAPPED PROBABILITY DENSITY (SI
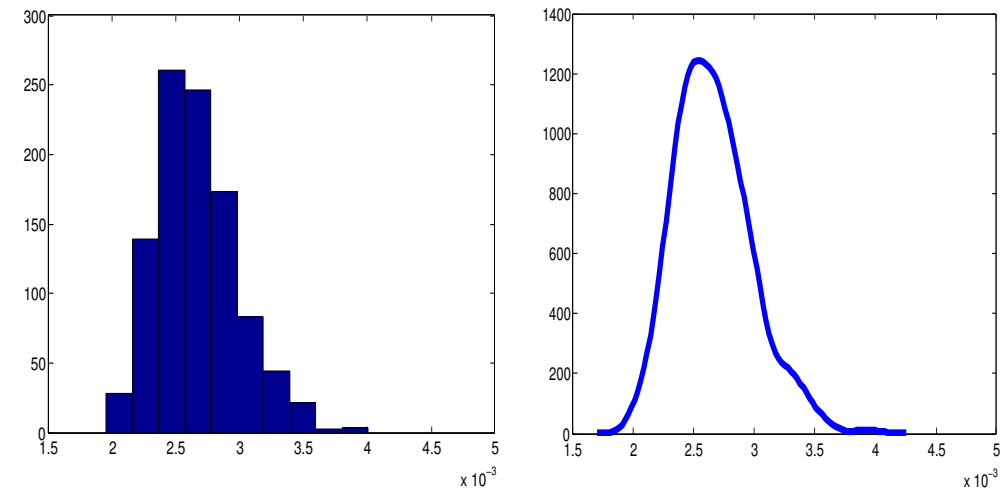

BAYESIAN BOOTSTRAPPED HISTOGRAM (SD)

BAYESIAN BOOTSTRAPPED PROBABILITY DENSITY (S[
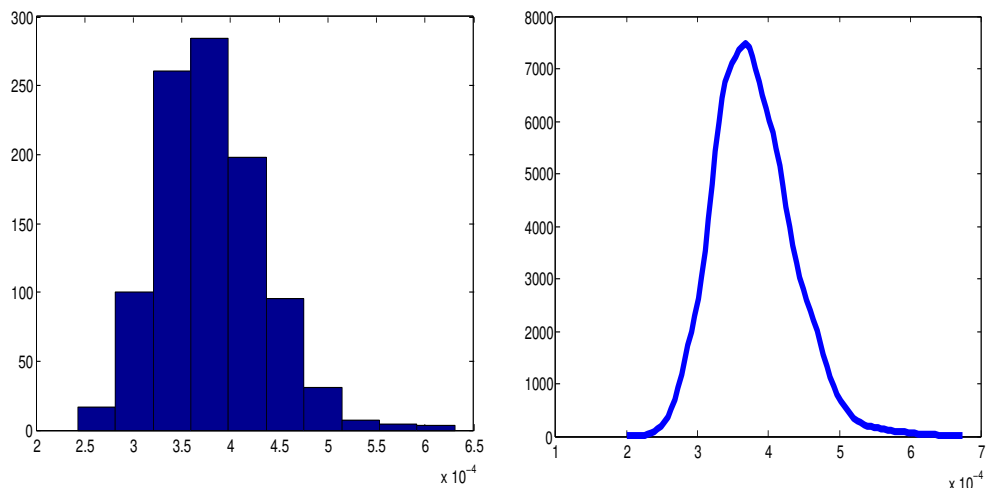

Fig. 9: Soft-data category. From 1000 bootstrap samples, spatially averaged SMAPEs histograms and probability densities are plotted, for trigonometric regression, combined with empirical-moment based classical (top), and Bayesian (bottom) residual prediction 


\section{Final comments}

One can observe the agreement between the respective performance-based model classification results, obtained from random $k$-fold cross-validation, and bootstrap estimation in Sections 5.1 and 5.2. In the hard-data category, the best performance is displayed by RBF and GP. Similar bootstrapping characteristics are observed for BNN and SVR, with slightly larger values of spatially averaged SMAPEs, reflected in the location of the mode, in the histograms and probability densities displayed in Figures 5 and 6 . These four regression methodologies show a similar degree of variability, regarding the spatially averaged SMAPEs sample values. A higher variability than RBF, GP, BNN and SVR is displayed by MLP spatially averaged SMAPEs bootstrap sample values. MLP bootstrapped mode is also slightly shifted to the right. The worst performance corresponds to GRNN (see also Table 6). In the soft-data category, where our approach is incorporated to the empirical comparative study, almost the same empirical ML model ranking holds. Some differences are found in the bootstrap confidence intervals, and histogram and probability densities computed. For instance, GRNN seems to be favored by soft-data category, while MLP displays worse performance in this category. Hence, smaller differences between GRNN and MPL are displayed in the soft-data category. A slightly improvement, in the soft-data category, of BNN relative to SVR is observed, preserving almost the same performance. RBF and GP display better performance in the soft-data category, being RFB a bit superior to GP in this category (see Table 10, and Figure 8). The trigonometric regression and classical multivariate time series residual prediction approach, based on the empirical moments, displays similar results to GRNN, with slightly better performance of GRNN, observed in the bootstrap intervals and histogram/probability density (see Figures 7 and 9). However, as given in Figures 8 and 9 the trigonometric regression and Bayesian residual prediction presents almost the same 'performance as BNN, with some slightly better probability distribution features of our approach respect to BNN (see also bootstrap intervals). Our approach is less affected by the random splitting of the sample, in the implementation of the random $k$-fold cross validation procedure, since a dynamical spatial residual model is fitted in a second (objective) step. Thus, the proposed multivariate time series classical and Bayesian regression residual modeling fits the short-term spatial linear correlations displayed by the softdata category. However, the price we pay for increasing model complexity is reflected in the resulting SMAPEs based random $k$-fold and bootstrap model classification results obtained.

The spatial component effect is reflected in Tables 6 (hard-data), where spatial heterogeneities displayed by random 10-fold cross-validation SMAPEs errors are observed (see also Table 3 in the Supplementary Material). While Table 7 (see also Table 4 in the Supplementary Material) reveals the benefits obtained in some of the ML regression models tested from soft-data information. Particularly, in this category, possible spatial linear correlations are incorporated to the analysis, in terms of soft-data, defined by the curve 
data projections onto the empirical eigenvectors, diagonalizing the spatial linear correlation structure.

\section{Declarations}

- Availability of data and material. The Supplementary Material uploaded contains the plots of the observed COVID-19 mortality cumulative cases curves, as well as of the corresponding COVID-19 mortality log-risk curves. The rest is not applicable.

- Competing interests. The author(s) declare(s) that they have no competing interests.

- Funding. This work has been supported in part by projects PGC2018099549-B-I00 of the Ministerio de Ciencia, Innovación y Universidades, Spain (co-funded with FEDER funds), and by grant A-FQM-345-UGR18 cofinanced by ERDF Operational Programme 2014-2020, and the Economy and Knowledge Council of the Regional Government of Andalusia, Spain. (In section Acknowledgements below, all sources of funding are also declared).

- Authors contributions. Not applicable

- Acknowledgements. The subject of this paper was originally developed, in a first stage, under the seminars hold in the Unidad de Transferencia del IMUS about Matemáticas y la COVID. We also thank the organizer, Professor Emilio Carrizosa.

(See also section Acknowledgements below).

\section{Acknowledgements}

This work has been supported in part by projects PGC2018-099549-B-I00 of the Ministerio de Ciencia, Innovación y Universidades, Spain (co-funded with FEDER funds), and by grant A-FQM-345-UGR18 cofinanced by ERDF Operational Programme 2014-2020, and the Economy and Knowledge Council of the Regional Government of Andalusia, Spain.

The subject of this paper was originally developed, in a first stage, under the seminars hold in the Unidad de Transferencia del IMUS about Matemáticas y la COVID. We also thank the organizer, Professor Emilio Carrizosa.

\section{References}

1. O. O. Aalen, O. Borgan and H. K. Gjessing (2008). Survival and event history analysis: a process point of view. Springer Science \& Business Media, New-York.

2. C. Abboud, O. Bonnefon, E. Parent and S. Soubeyrand (2019). Dating and localizing an invasion from post-introduction data and a coupled reaction-diffusion-absorption model. Journal of Mathematical Biology 79, 765-789.

3. C. Agostinelli (2001). Robust model selection in regression via weighted likelihood methodology. Statistics \& Probability Letters 56, 289-300. 
4. E. Alpaydin (2004). Introduction to Machine Learning. MIT Press, Cambridge, MA.

5. H. Anderson and T. Britton (2000.). Stochastic epidemic models and their statistical analysis. Springer-Verlag, New-York.

6. J. Angulo, H.-L. Yu, A. Langousis, A. Kolovos, J. Wang, A. E. Madrid and G Christakos (2013). Spatiotemporal infectious disease modeling: A BME-SIR Approach. PLoS One 8(9): e72168.

7. M. Barstugan, U. Ozkaya and S. Ozturk (2020). Coronavirus (COVID-19) classification using ct images by machine learning methods. arXiv preprint arXiv:2003.09424

8. E. E. Beretta, T. Hara, W. Ma and Y. Takeuchi (2001). Global asymptotically stability of an SIR epidemic model with distributed time delay. Nonlinear Anal Theory Methods Appl 47, 4107-4115.

9. R. Blanquero, E. Carrizosa, M. A. Jiménez-Cordero and B. Martín-Barragán (2020). Selection of time instants and intervals with support vector regression for multivariate functional data. Computers \&f Operations Research 123 10.1016/j.cor.2020.105050.

10. B. M. Bolker and B. Grenfell (1996). Impact of vaccination on the spatial correlation and persistence of measles dynamics. Proceedings of the National Academy of Sciences 93, $12648-12653$.

11. D. Bosq (2000). Linear processes in function spaces. Lecture notes in statistics 149. Springer, New-York.

12. D. Bosq and M. D. Ruiz-Medina (2014). Bayesian estimation in a high dimensional parameter framework. Electron J Statist 8, 1604-1640.

13. D. L. Chao, J. D. Bloom, B. F. Kochin, R. Antia and I. M. Longini (2012). The global spread of drug-resistant influenza. Journal of the Royal Society Interface 9, 648-656.

14. O. Chapelle, V. Vapnik and Y. Bengio (2002). Model selection for small sample regression. Machine Learning 48, 9-23.

15. L.-Ch. Chien and L.-W. Chen (2020). Meteorological impacts on the incidence of COVID-19 in the U.S. Stoch Environ Res Risk Assess 34, 1675-1680.

16. G. Christakos, P. Bogaert and M. L. Serre (2002). Advanced functions of temporal GIS, Springer-Verlag, New York, N.Y.

17. Z. Du, X. Xu, Y. Wu, L. Wang, L. A. Cowling and B. J. Meyers (2020). Serial interval of COVID-19 among publicly reported confirmed cases. Emerg. Infect. Dis. 26(6).

18. J. Dushoff, J. Plotkin, S. Levin and D. Earn (2004). Dynamical resonance can account for seasonality of influenza epidemics. Proceedings of the National Academy of Sciences of the United States of America 101, 16915-16916.

19. M. Elhia, A. Laaroussi, M. Rachik, Z. Rachik and E. Labriji (2014). Global stability of a susceptible-infected-recovered (SIR) epidemic model with two infectious stages and treatment. Int J Sci Res 3, 114-121.

20. T. R. Fleming and D. P. Harrington (1991). Counting processes and survival analysis. Wiley Series in Probability and Mathematical Statistics: Applied Probability and Statistics. John Wiley \& Sons, Inc., New-York.

21. L. N. Guin and P. K. Mandal (2014). Spatiotemporal dynamics of reaction-diffusion models of interacting populations. Appl Math Model 38, 4417-4427.

22. T. Hastie, R. Tibshirani and J. Friedman (2001). The elements of statistical learning. Springer Series in Statistics. Springer-Verlag, New-York.

23. He J, Chen G, Jiang Y, Jin R, Shortridge A, Agusti S, Hea M, Wua J, Duarte CM, Christakos G (2020) Comparative infection modeling and control of COVID-19 transmission patterns in China, South Korea, Italy and Iran. Science of the Total Environment 747

24. A. Huppert and G. Katriel (2013). Mathematical modelling and prediction in infectious disease epidemiology. Clin Microbiol Infect 19, 999-1005.

25. A.V. Ivanov, N.N. Leonenko, M.D. Ruiz Medina, and B.M. Zhurakovsky (2015). Estimation of harmonic component in regression with cyclically dependent errors. Stastics: A Journal of Theoretical and Applied Statistics 49, 156-186.

26. B. Ivorra, M. R. Ferrández, M. Vela-Pérez and A. M. Ramos (2020). Mathematical modeling of the spread of the coronavirus disease 2019 (COVID-19) taking into account the undetected infections. The case of China. Commun Nonlinear Sci Numer Simulat 88, 105-303.

27. B. Ivorra, A. M. Ramos and D. Ngom (2015). Be-CoDiS: A mathematical model to predict the risk of human diseases spread between countries. Validation and application to the 2014 ebola virus disease epidemic. Bull Math Biol 77, 1668-1704. 
28. C. Ji, D. Jiang and N. Shi (2012). The behavior of an SIR epidemic model with stochastic perturbation. Stoch Anal Appl. 30, 755-773.

29. M. J. Keeling, D. A. Rand and A. J. Morris (1997). Correlation models for childhood epidemics. Proceedings of the Royal Society of London 264, 1149-1156.

30. M. J. Keeling and P. Rohani (2008). Modeling infectious diseases in humans and animals. Princeton University Press, Princeton.

31. W. Kermack and A. McKendrick (1927). Contributions to the mathematical theory of epidemics - I. Proceedings of the Royal Society of Edinburgh A 115, 700-721.

32. M. A. Khan and A. Atangana (2020). Modeling the dynamics of novel coronavirus (2019-nCov) with fractional derivative. Alex. Eng. J.. doi.org/10.1016/j.aej.2020.02.033.

33. A. J. Kucharski, T. W. Russell, C. Diamond, Y. Liu, J. Edmunds and S. Funk et al. (2020). Early dynamics of transmission and control of COVID-19: a mathematical modelling study. Lancet Infect Dis. doi.org/10.1016/S1473-3099(20)30144-4.

34. Y. A. Kuznetsov and C. Piccardi (1994). Bifurcation analysis of periodic SEIR and SIR epidemic models. J Math Biol 32, 109-121.

35. A. E. Laaroussi, M. Rachik and M. Elhia (2018). An optimal control problem for a spatiotemporal SIR model Int. J. Dynam. Control 6, 384-397.

36. A. Langousis and A. A. Carsteanu (2020). Undersampling in action and at scale: application to the COVID-19 pandemic. Stoch Environ Res Risk Assess 34, 1281-1283.

37. C. Malesios, N. Demiris, P. Kostoulas, K. Dadousis, T. Koutroumanidis and Z. Abas (2016). Spatio-temporal modelling of foot-and-mouth disease outbreaks. Epidemiol. Infect. 144, 2485-2493.

38. C. C. McCluskey (2010). Complete global stability for an SIR epidemic model with delay distributed or discrete. Nonlinear Anal Real World Appl 11, 55-59.

39. F. A. Milner and R. Zhao (2008). SIR model with directed spatial diffusion. Math Popul Stud 15, 160-181.

40. M. Mohammady, H. Reza Pourghasemi, M. Amiri and J. P. Tiefenbacher (2021). Spatial modeling of susceptibility to subsidence using machine learning techniques. https://doi.org/10.1007/s00477-020-01967-x

41. H. Nishiura, N. M. Linton and A. R. Akhmetzhanov (2020). Serial interval of novel coronavirus (COVID-19) infections. Int. J. Infect. Dis. 93, 284-286.

42. D. Pak, K. Langohr, J. Ning, J. Cortés Martínez, G. Gómez-Melis and Y. Shen (2020). Modeling the coronavirus disease 2019 incubation period: impact on quarantine policy doi.org/10.1101/2020.06.27.20141002.

43. S. Pathak, A. Maiti and G. Samanta (2010). Rich dynamics of an SIR epidemic model. Nonlinear Anal Model Control 15, 71-81.

44. A.M. Ramosa, M.R. Ferrández, M. Vela-Pérez and B. Ivorra (2020). A simple but complex enough $\theta$-SIR type model to be used with COVID-19 real data. Application to the case of Italy. doi.org/10.13140/RG.2.2.32466.17601.

45. A. Remuzzi and G. Remuzzi (2020). COVID-19 and Italy: what next? The Lancet. doi.org/10.1016/S0140-6736(20)30690-5

46. K. Roosa, Y. Lee, R. Luo, A. Kirpich, R. Rothenberg, J. Hyman et al. (2020). Real-time forecasts of the COVID-19 epidemic in China from February 5th to February 24th. Infect Dis Modell 5, 256-263.

47. L. Roques and O. Bonnefon (2016). Modelling population dynamics in realistic landscapes with linear elements: A mechanistic-statistical reaction-diffusion approach. PloS One 11(3): 0151217.

48. L. Roques, S. Soubeyrand and J. Rousselet (2011). A statistical-reaction-diffusion approach for analyzing expansion processes. J Theor Biol. 274, 43-51.

49. M. Sekiguchi and E. Ishiwata (2010). Global dynamics of a discretized SIRS epidemic model with time delay. J Math Anal Appl 371, 195-202.

50. B. Sivakumar (2020). COVID-19 and water. Stoch Environ Res Risk Assess. https://doi.org/10.1007/s00477-020-01837-6

51. R. Sujath, J. M. Chatterjee and A. E.Hassanien (2020). A machine learning forecasting model for COVID-19 pandemic in India. Stoch Environ Res Risk Assess. 34, 959-972.

52. R. Sujath, J. M. Chatterjee and A. E.Hassanien (2020). Correction to: A machine learning forecasting model for COVID-19 pandemic in India. Stoch Environ Res Risk Assess. https://doi.org/10.1007/s00477-020-01843-8 
53. Y. Takano and R. Miyashiro (2020). Best subset selection via cross-validation criterion. TOP 28, 475-488.

54. E. Tornatore, S. M. Buccellato and P. Vetro (2005). Stability of a stochastic SIR system. Phys A Stat Mech Its Appl 354, 111-126.

55. A. Torres, M. P. Frías and M. D. Ruiz-Medina (2016). Log-Gaussian Cox processes in infinite-dimensional spaces. Theor Prob Math Stat 95, 157-177.

56. E. Volz (2008). SIR dynamics in random networks with heterogeneous connectivity. Journal of Mathematical Biology 56, 293-310.

57. C. Wang, P. W. Horby, F. Hayden and G. F. Gao (2020). A novel coronavirus outbreak of global health concern. Lancet 395, 470-473.

58. R. K. Wasiur, B. Choiy, E. Kenahz and G. A. Rempa (2019). Survival dynamical systems for the population-level analysis of epidemics. arXiv.1901.00405.

59. G. Webb (1981). A reaction-diffusion model for a deterministic diffusive epidemic. $J$ Math Anal Appl 84, 150-161.

60. Y. Xu, L. Allena and A. Perelson (2007). Stochastic model of an influenza epidemic with drug resistance. Journal of Theoretical Biology 248, 179-193.

61. J. Yu, D. Jiang and N. Shi (2009). Global stability of two-group SIR model with random perturbation. J Math Anal Appl. 360 235-244.

62. Wb. Zhang, Y. Ge, M. Liu et al. (2020) Risk assessment of the step-by-step return-towork policy in Beijing following the COVID-19 epidemic peak. Stoch Environ Res Risk Assess. https://doi.org/10.1007/s00477-020-01929-3

63. F. Zhang, Z. Li and F. Zhang (2008). Global stability of an SIR epidemic model with constant infectious period. Appl Math Comput. 199, 285-291.

64. T. Zhou, Z. Fu and B. Wang (2006). Epidemic dynamics on complex networks. Progress in Natural Science 16, 452-457.

65. F. Zhou, T. Yu, R. Du, G. Fan, Y. Liu, Z. Liu, J. Xiang, Y. Wang, B. Song, X. Gu, et al (2020). Clinical course and risk factors for mortality of adult inpatients with COVID19 in Wuhan, China: a retrospective cohort study. The Lancet. doi.org/10.1016/S0140$6736(20) 30566-3$ 
Figures
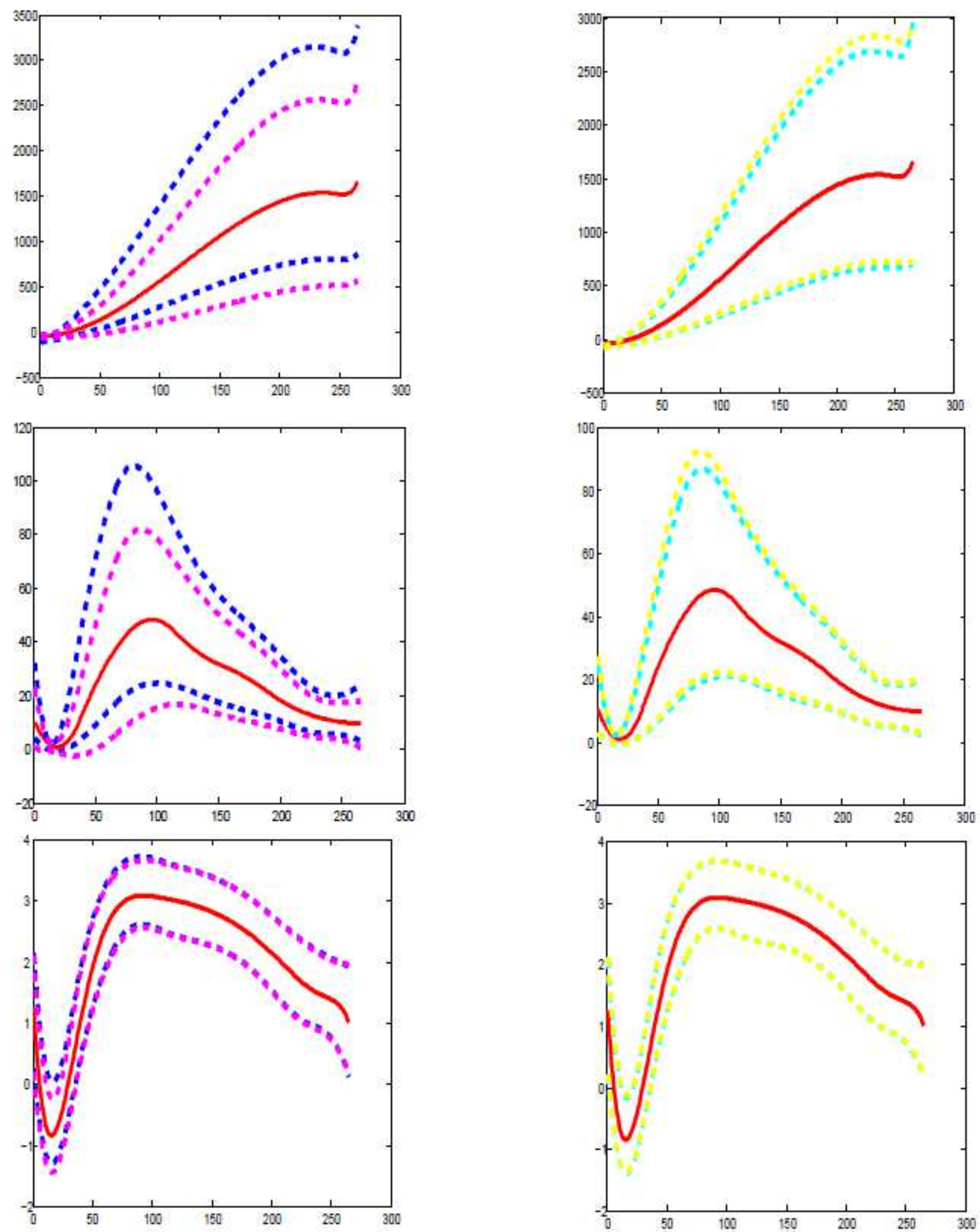

\section{Figure 1}

At the top, COVID $\{19$ mortality mean cumulative curve in Spain, since March, 8, 2020 to May, 13, 2020 (continuous red line, 265 temporal nodes), and bootstrap curve confidence intervals, at the left-hand-side, 11 (dashed blue lines) and I2 (dashed magenta lines), and at the right-hand-side, I3 (dashed green lines) 
and 14 (dashed yellow lines). Plots at the center and bottom reflect the same information respectively referred to the mean intensity (spatial averaged COVID $\{19$ mortality risk curve), and log-intensity (spatial averaged COVID $\{19$ mortality log-risk curve) curves in Spain. All the confidence bootstrap intervals are computed at confidence level $1-a=0: 95$; from 1000 bootstrap samples

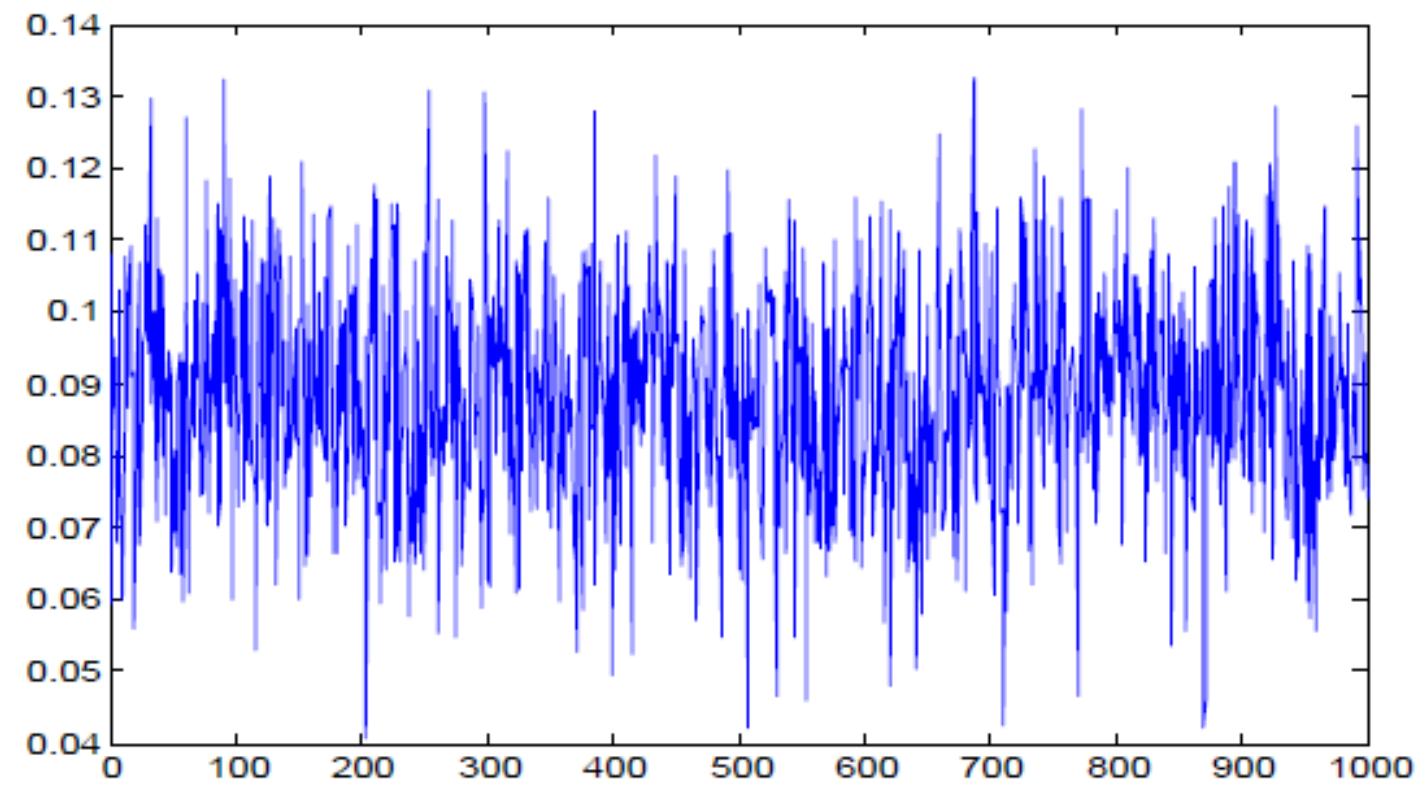

BOOTSTRAP HISTOGRAM

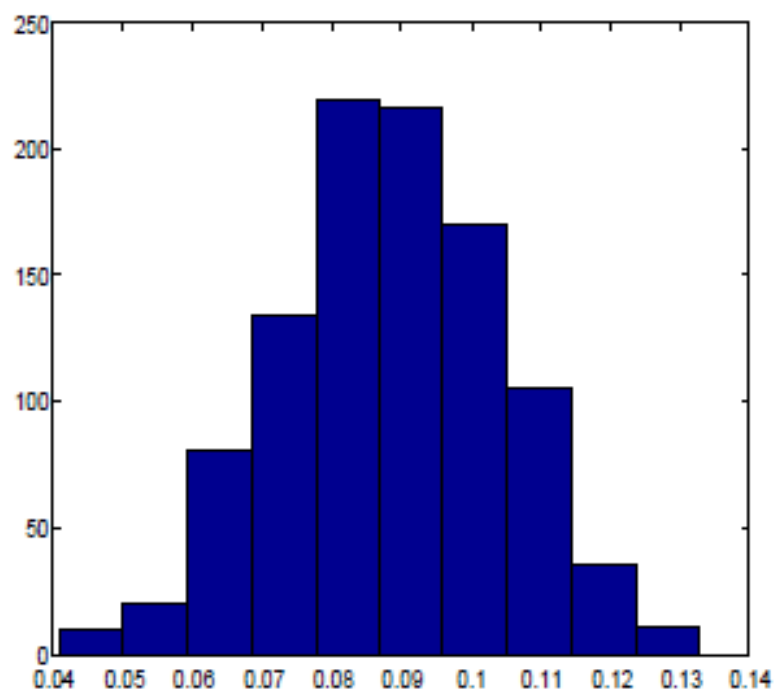

BOOTSTRAP PROBABILITY DENSITY

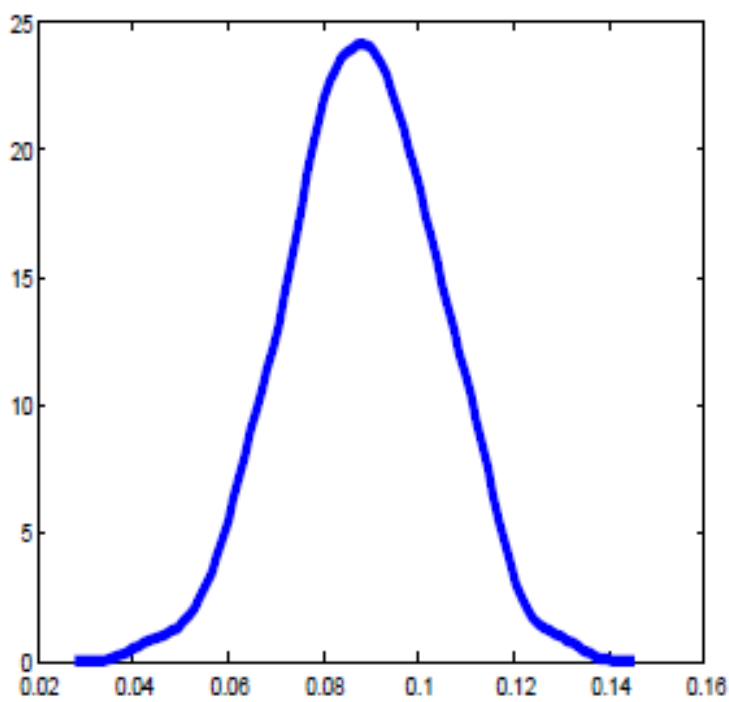

\section{Figure 2}

At the top, sample values of the spatial mean of the minimized empirical risk in the trigonometric regression. Bootstrap histogram (left-hand side-bottom), and bootstrap probability density (right-hand- 
side-bottom) of the spatial mean of the minimized regression empirical risk, based on 1000 bootstrap samples

17X17=289 EMPIRICAL PROJECTIONS OF RHO

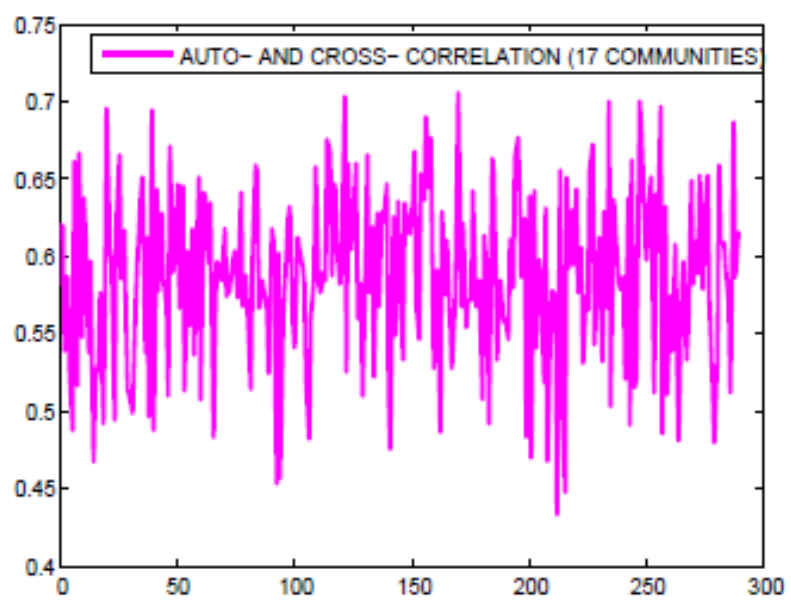

THEORETICAL AND EMPIRICAL PRIOR

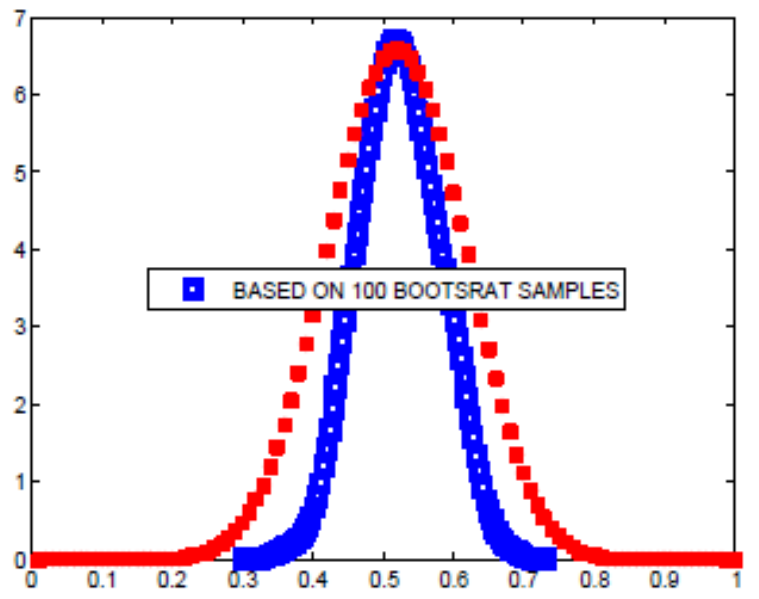

\section{Figure 3}

At the left $\{$ hand side, empirical projections of the autocorrelation operator p; reflecting temporal autocorrelation and cross-correlation between the 17 Spanish Communities analyzed. At the right-hand side, the considered prior probability density (red squares) of a scaled, by factor $1=3$; Beta distributed random variable with shape parameters 14 and 13 is compared with the bootstrap fitting of an empirical prior (blue squares) 

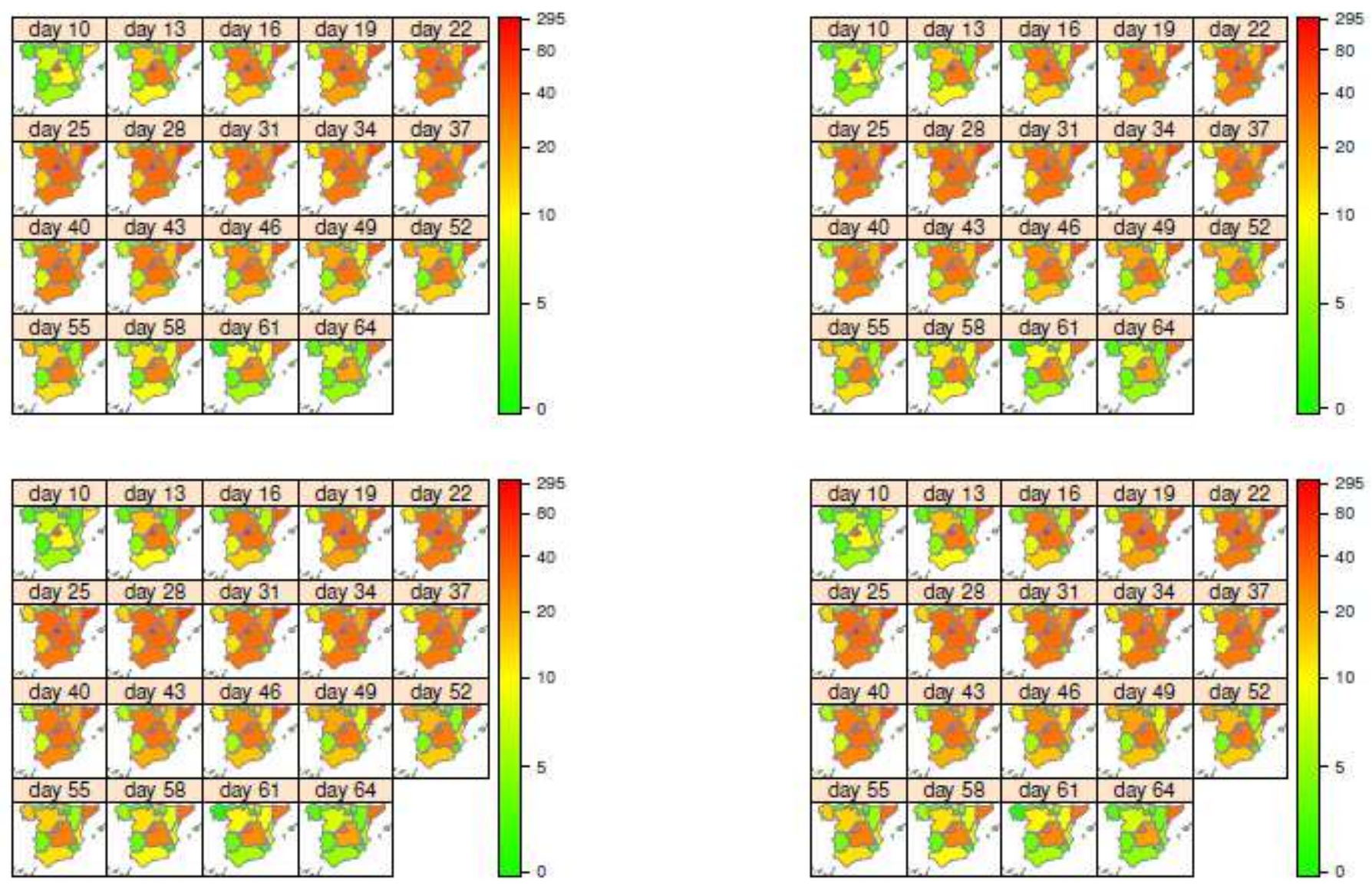

\section{Figure 4}

COVID $\{19$ mortality risk maps, since March, 8 to May, 13, 2020. Observed (left- hand-side) and estimated (right-hand side) maps, computed from trigonometric regression, combined with classical (first line) and Bayesian (second line) residual predictors 
BOOTSTRAPPED HISTOGRAM GEN

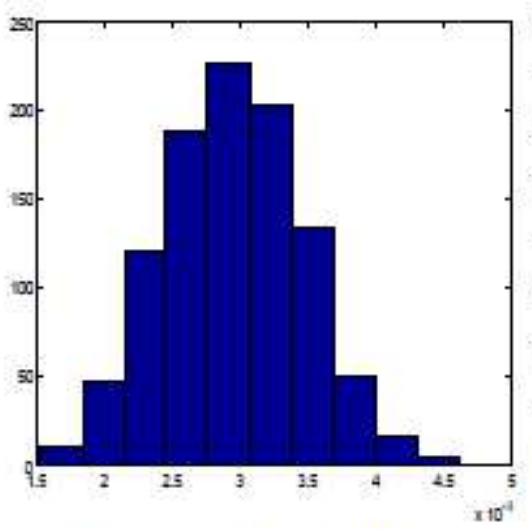

BOOTSTRAPPED HISTOGRAM MVP

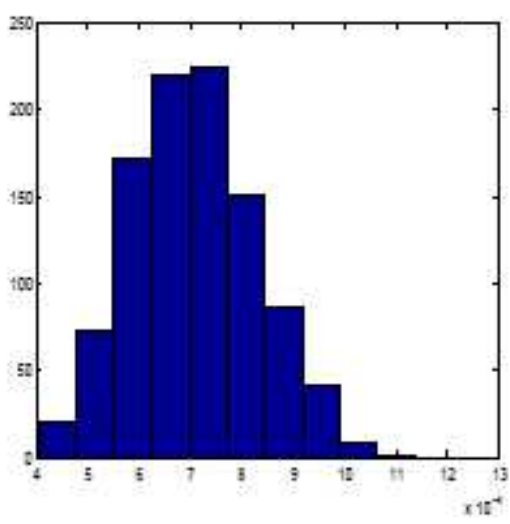

BOOTSTRAPPED HISTOGRAM SUR

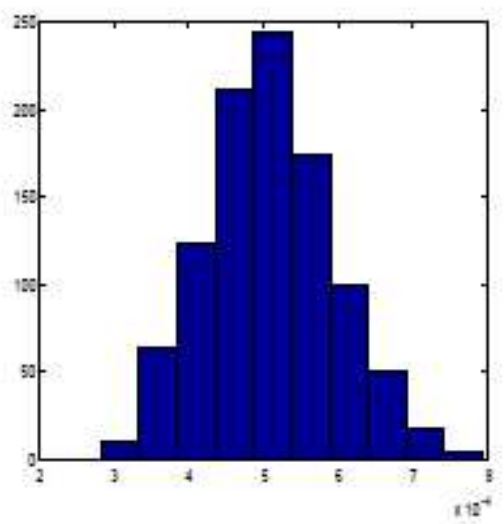

BOOTSTRAPPED PROBABILITY DENSITY GRIN

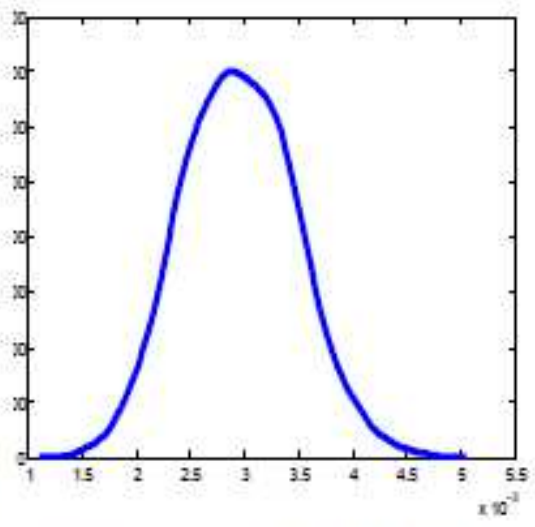

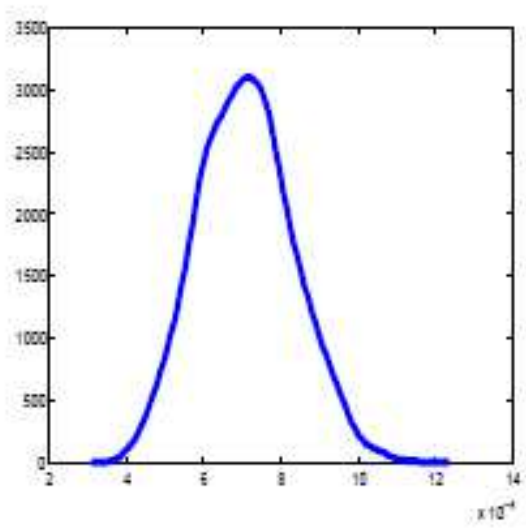
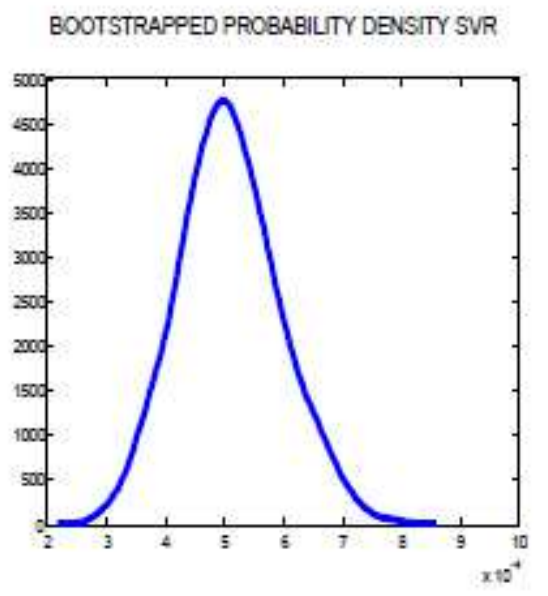

Figure 5

Hard-data category. From 1000 bootstrap samples, spatially averaged SMAPEs histograms and probability densities are plotted, for GRNN (top), MLP (center), and linear SVR (bottom) 


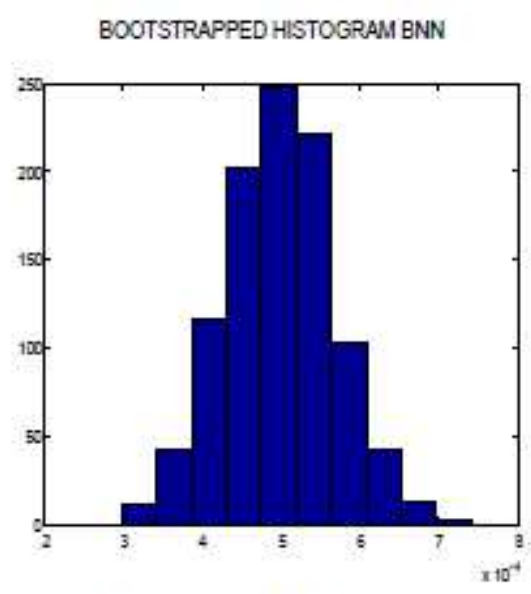

BOOTSTRAPPED HISTOGRAM RBF
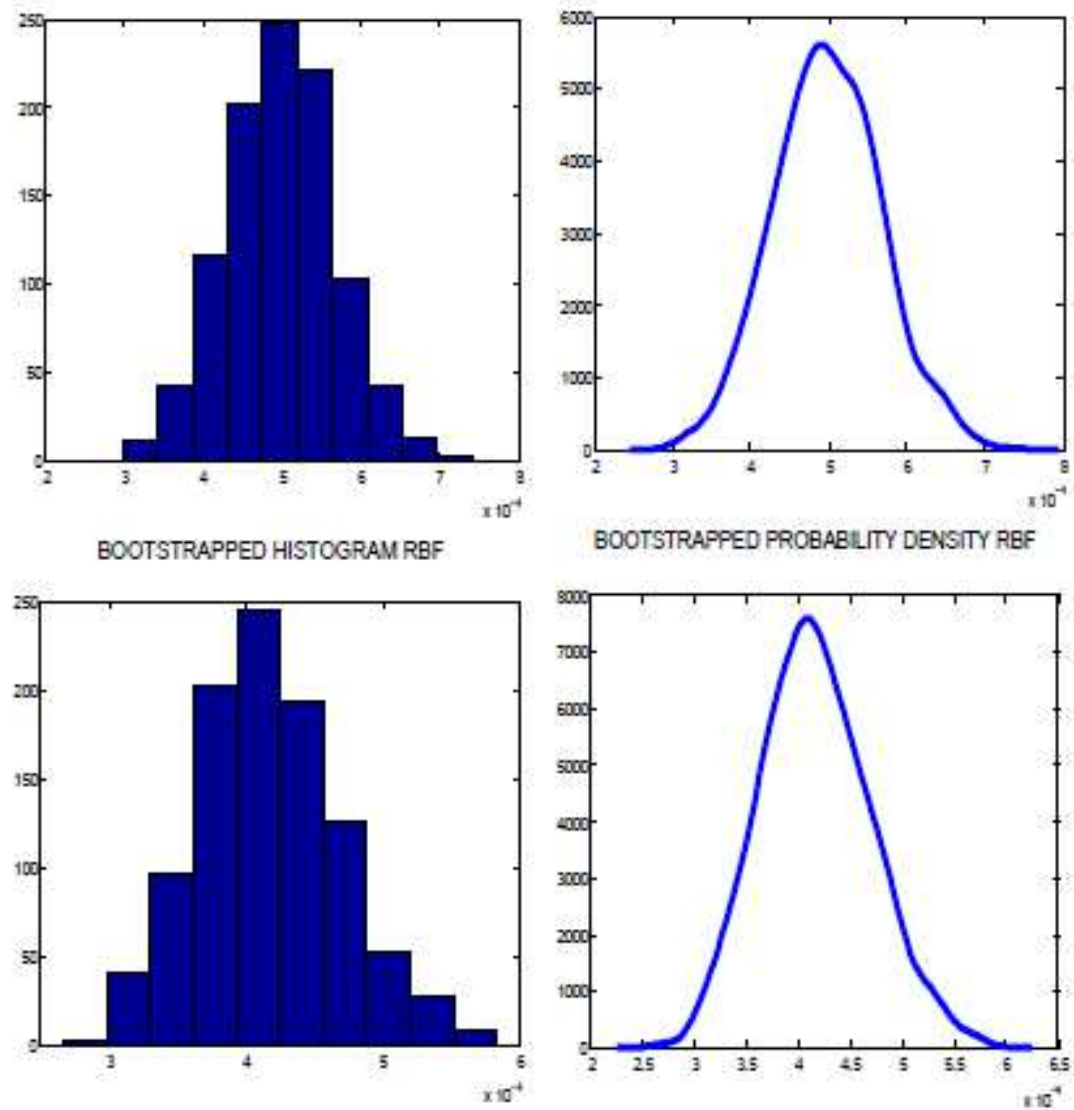

BOOTSTRAPPED PROBABLITY DENSTYY RBF

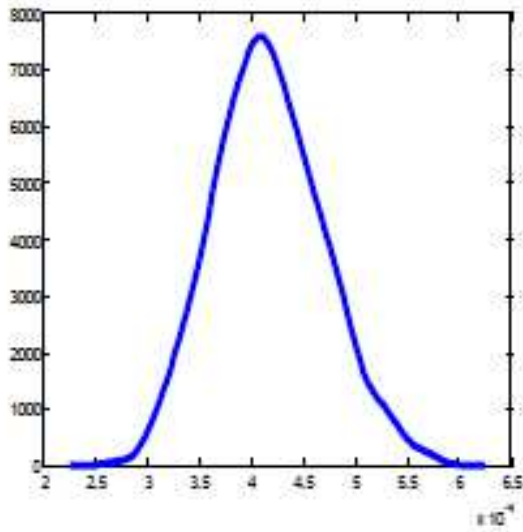

BOOTSTRAPPED HISTOGRAM GP

BOOTSTRAPPED PROBABIUTY DENSTYY GP
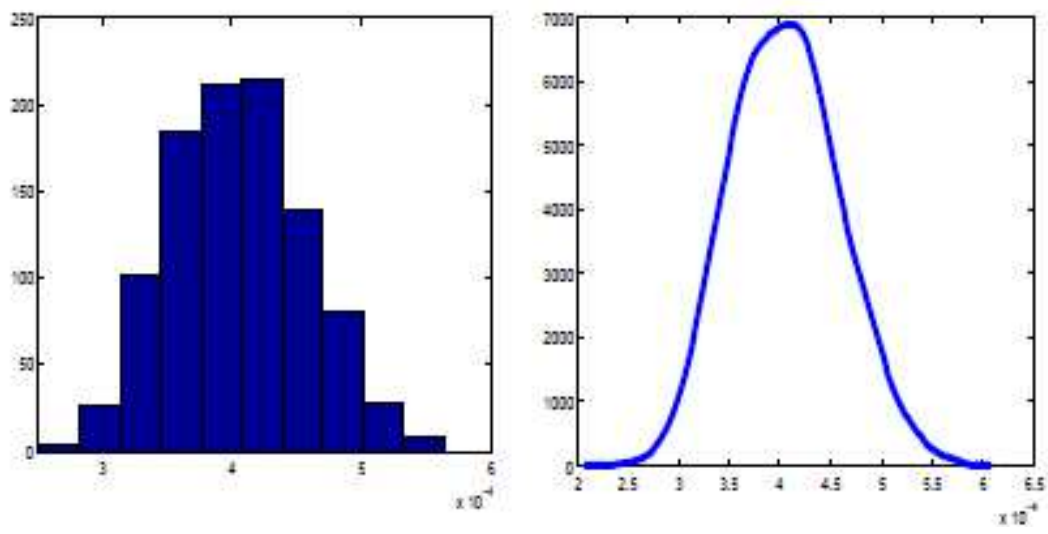

Figure 6

Hard-data category. From 1000 bootstrap samples, spatially averaged SMAPEs histograms and probability densities are plotted, for BNN (top), RBF (center), and GP (bottom) 
BOOTSTRAPPED HISTOGRAM GRNN (SD)

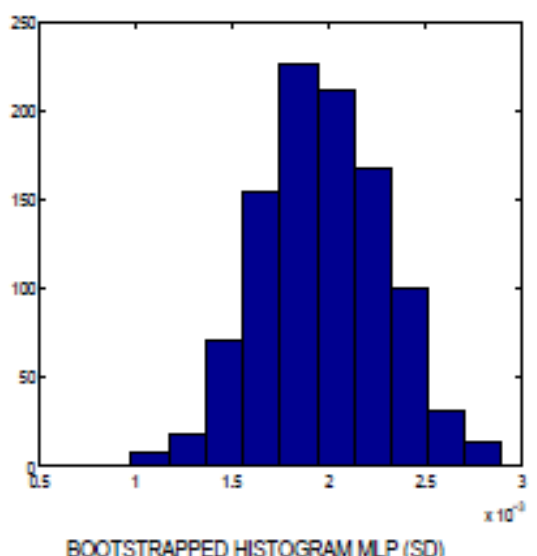

BOOTSTRAPPED HISTOGRAM MP (SD)

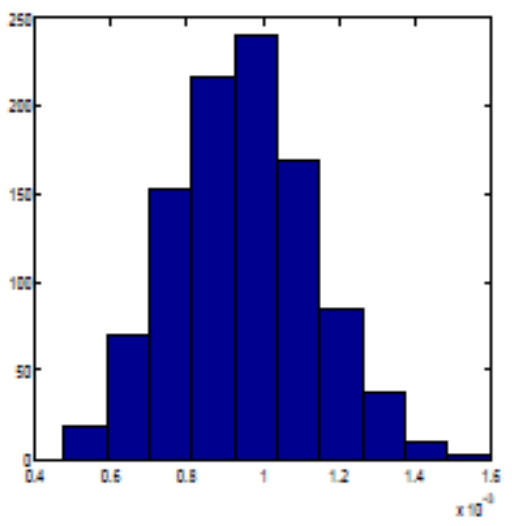

BOOTSTRAPPED HISTOGRAM SVR (SD)

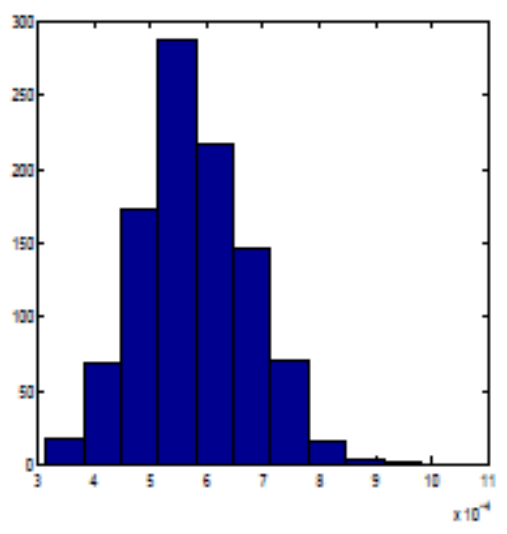

BOOTSTRAPPED PROBABILITY DENSITY GRNN (SD)

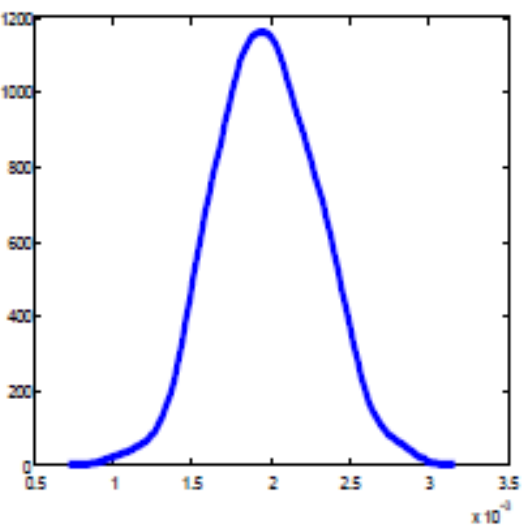

BOOTSTRAPPED PROBABILITY DENSTTY MP (SD)

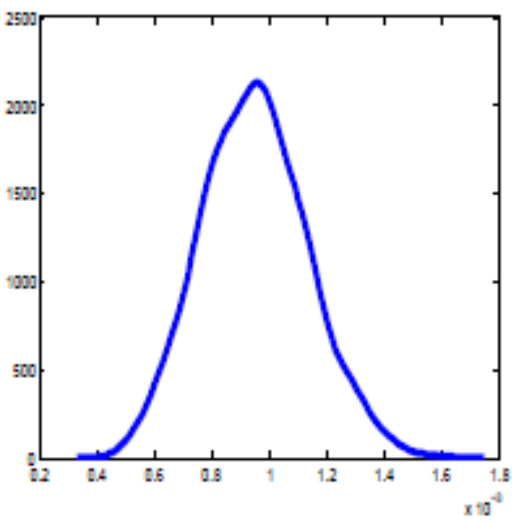

BOOTSTRAPPED PROBABILITY DENSITY SVR (SD)

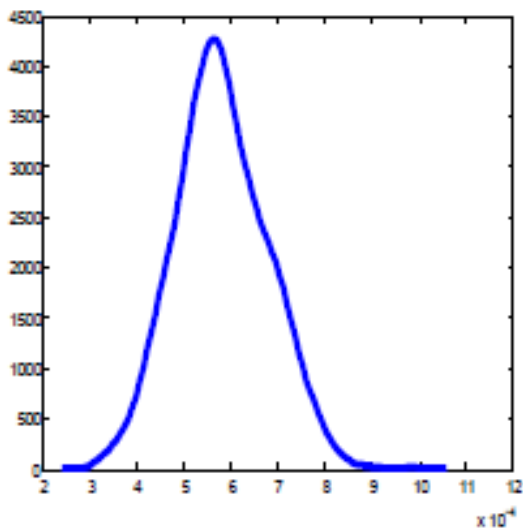

Figure 7

Soft-data category. From 1000 bootstrap samples, spatially averaged SMAPEs histograms and probability densities are plotted, for GRNN (top), MLP (center) and non- linear SVR (bottom) 
BOOTSTRAPPED HISTOGRAM ENN (SD)

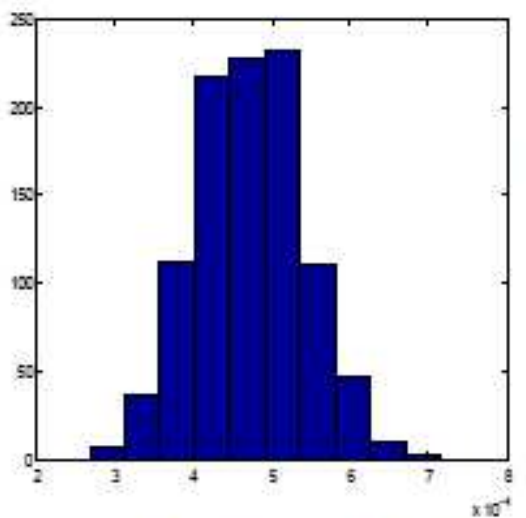

BOOTSTRAPPED HISTOGRAM RBF (SD)

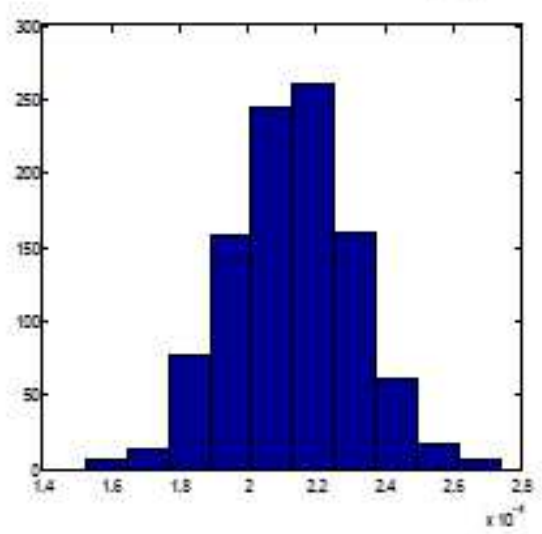

BCOTSTRAPPED HISTOGRAM GP (SD)

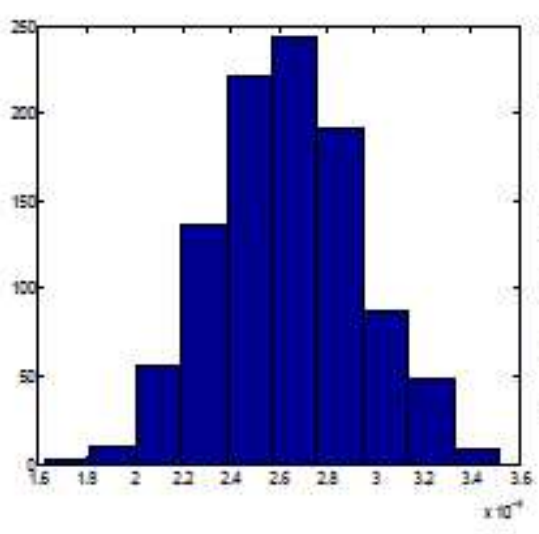

BOOTSTRAPPED PROBABIUTY DENSTYY BNN (SD)

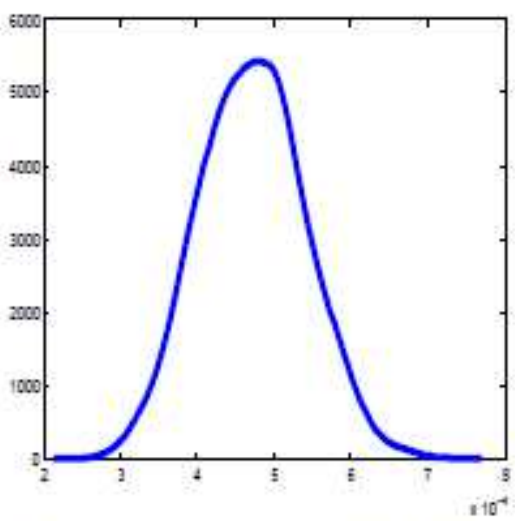

BOOTSTRAPPED PROBABILITY DENSITY RBF (SD)

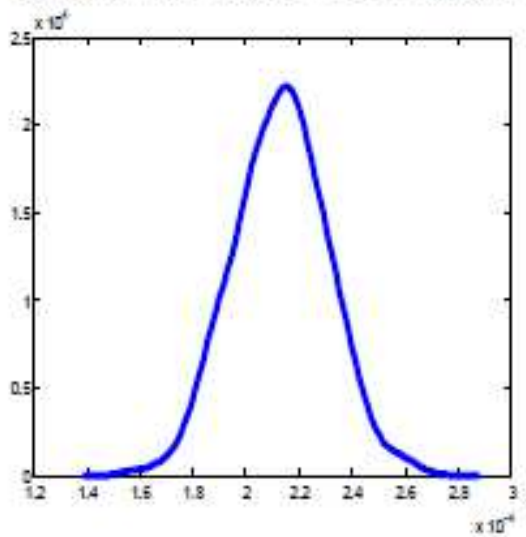

BOOTSTRAPPED PROBABILITY DENSITY GP (SD)

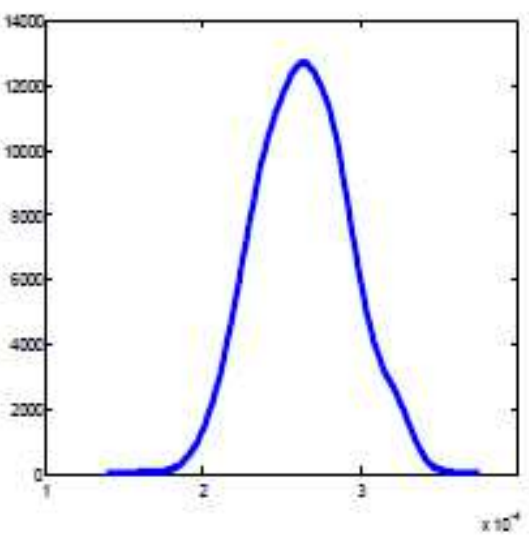

Figure 8

Soft-data category. From 1000 bootstrap samples, spatially averaged SMAPEs histograms and probability densities are plotted, for BNN (top), RBF (center) and GP (bottom) 


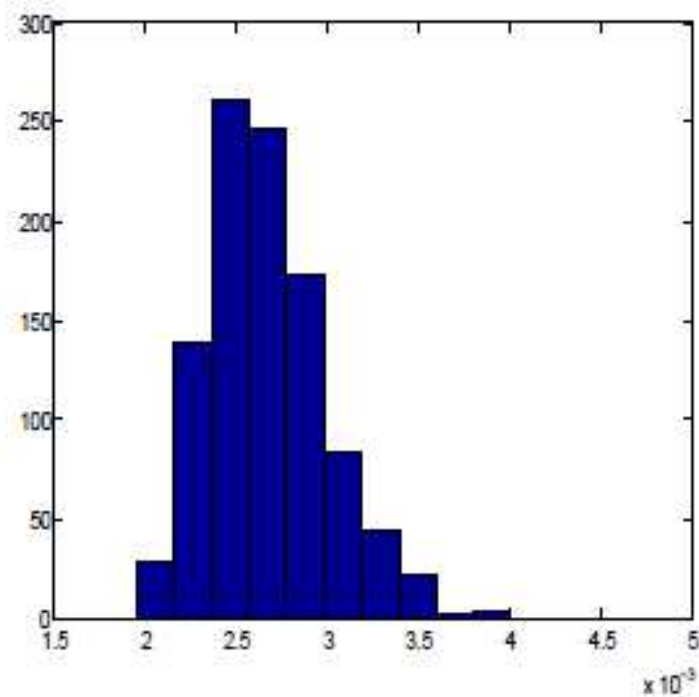

BAYESIAN BOOTSTRAPPED HISTOGRAM (SD)

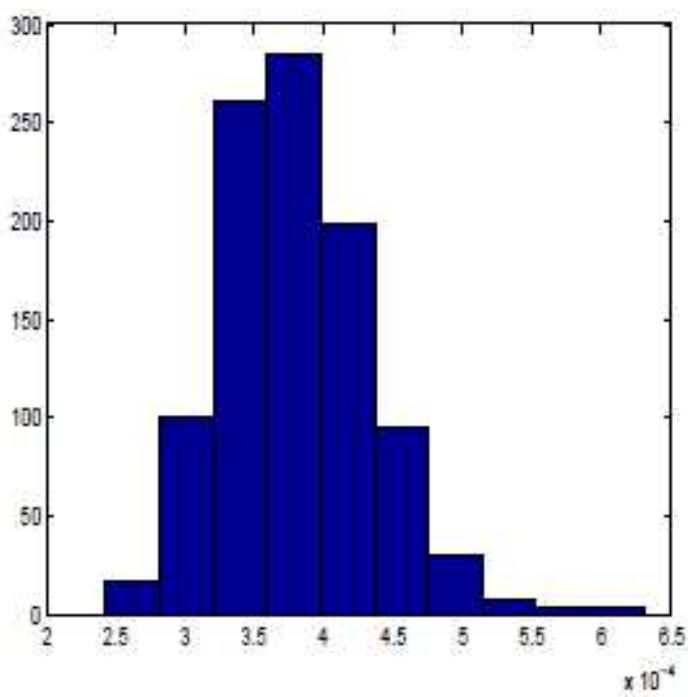

CLASSICAL BOOTSTRAPPED PROBABILITY DENSITY (SI

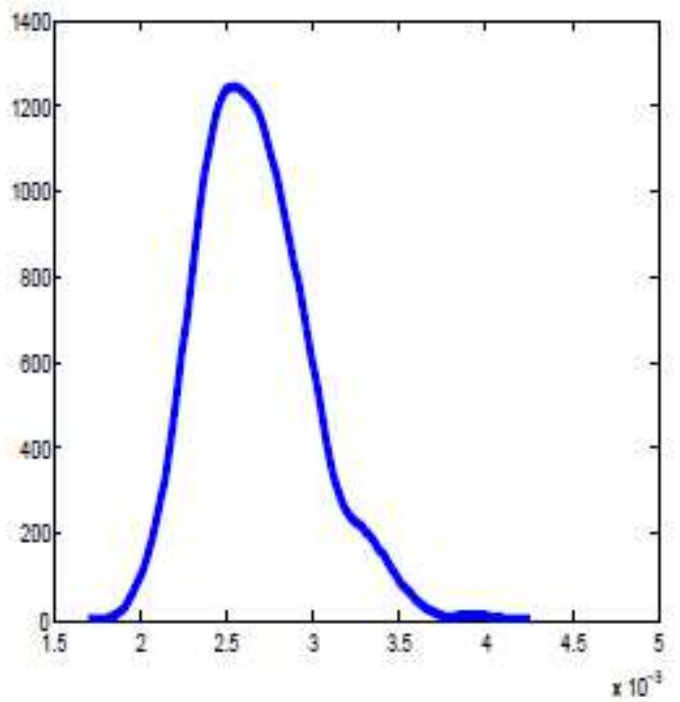

BAYESIAN BOOTSTRAPPED PROBABILITY DENSITY (SC

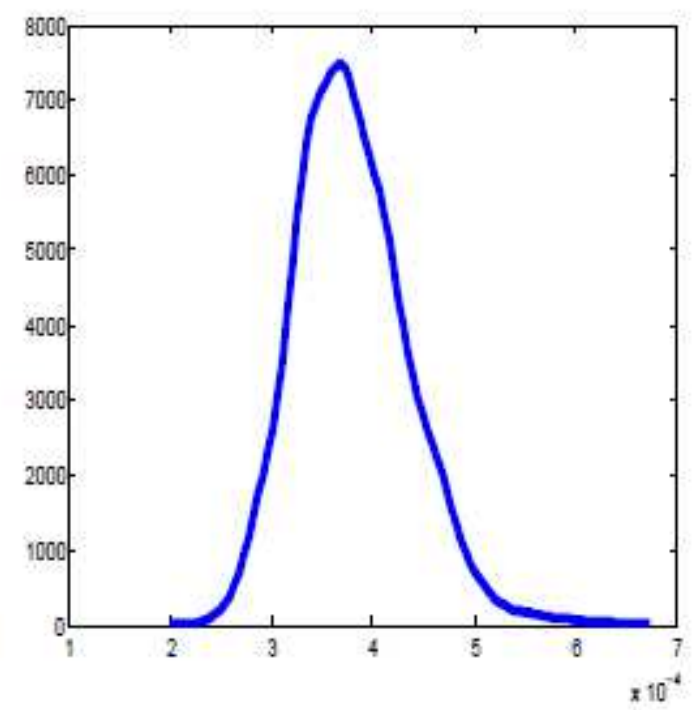

\section{Figure 9}

Soft-data category. From 1000 bootstrap samples, spatially averaged SMAPEs histograms and probability densities are plotted, for trigonometric regression, combined with empirical-moment based classical (top), and Bayesian (bottom) residual prediction

\section{Supplementary Files}

This is a list of supplementary files associated with this preprint. Click to download.

- SupplementaryMaterial16012021.pdf 